\title{
Universality in the Thomas-Fermi- von Weizsäcker Model of Atoms and Molecules
}

Jan Philip Solovej ${ }^{\star \star \star \star}$

Department of Mathematics, Princeton University, Princeton, NJ 08544, USA

\begin{abstract}
We study the Thomas-Fermi-von Weizsäcker theory of atoms and molecules. The main result is to prove universality of the structure of very large atoms and molecules, i.e., proving that the structure converges as the nuclear charges go to infinity. Furthermore we uniquely characterize the limit density as the solution to a renormalized TFW-equation. This is achieved by characterizing the strong singularities of solutions to the non-linear TFWsystem.
\end{abstract}

\section{Introduction}

The Thomas-Fermi-von Weizsäcker (TFW) theory for a molecule of $K$ nuclei at positions $\mathscr{R}_{1}, \ldots, \mathscr{R}_{K} \in \mathbb{R}^{3}$ and with nuclear charges $z_{1}, \ldots, z_{K} \in \mathbb{R}_{+}$is defined by the energy functional

$$
\begin{aligned}
\mathscr{E}(\psi ; \underline{z} ; \mathscr{R})= & A \int_{\mathbb{R}^{3}}(\nabla \psi(x))^{2} d x+\frac{3}{5} \gamma \int_{\mathbb{R}^{3}}\left(\psi(x)^{2}\right)^{5 / 3} d x \\
& -\sum_{j=1}^{K} z_{j} \int_{\mathbb{R}^{3}} \psi(x)^{2}\left|x-\mathscr{R}_{j}\right|^{-1} d x+D\left(\psi^{2}, \psi^{2}\right),
\end{aligned}
$$

where

$$
D(f, g)=\frac{1}{2} \int_{\mathbb{R}^{3} \times \mathbb{R}^{3}} f(x)^{2}|x-y|^{-1} g(y)^{2} d x d y .
$$

Here $\underline{z}=\left(z_{1}, \ldots, z_{K}\right)$ and $\mathscr{R}=\left(\mathscr{R}_{1}, \ldots, \mathscr{R}_{K}\right) . \mathscr{E}$ is defined on the set

$$
G=\left\{\psi \in L^{10 / 3}\left(\mathbb{R}^{3}\right) \mid \psi \text { real, } \nabla \psi \in L^{2}, D\left(\psi^{2}, \psi^{2}\right)<\infty\right\} .
$$

\footnotetext{
* Supported by a Danish Research Academy Fellowship and U.S. National Science Foundation grant PHY-85-15288-A03

$\star \star$ Address from September 1989: Department of Mathematics, The University of Michigan, Ann Arbor, Michigan 48109-1003, USA
} 
On $G,|\mathscr{E}(\psi)|<\infty$. This and all the following statements about the TFW theory are proved in Lieb [10, Sect. VII] in which the foundation of the TFW-theory is established, see also Benguria et al. [1].

The first two terms in $\mathscr{E}$ represent the kinetic energy of the electrons in the molecule. The nuclei are considered infinitely heavy so they do not contribute to the kinetic energy. The third term is the nuclear attraction while the last term is the electronic repulsion. We are using units in which $h^{2}(2 m)^{-1}=-e=1$, where $e$ and $m$ are the charge and mass of the electron. The unit of length is $\frac{1}{2} a_{0}=\hbar^{2}(2 m)^{-1} e^{2}$, where $a_{0}$ is the Bohr radius.

The physically correct value for $\gamma$ is given by the Thomas-Fermi (TF) theory, $\gamma=\left(3 \pi^{2}\right)^{2 / 3}$, (the TF theory corresponds to $A=0$ above, see again [10]). The constant $A$ is usually chosen so as to reproduce the Scott term in the asymptotic $Z$-expansion of the true quantum energy (see $[10$, Theorem 7.30$]$ and $[7,15,16])$. The numerical value is $A=0.1859$ (see [8]).

By rescaling $\psi(x) \rightarrow A^{3 / 2} \gamma^{-3} \psi\left(A^{1 / 2} \gamma^{-3 / 2} x\right), \mathscr{E} \rightarrow A^{7 / 2} \gamma^{-9 / 2} \mathscr{E}, \underline{z} \rightarrow A^{3 / 2} \gamma^{-3 / 2} \underline{\underline{E}}$ and $\mathscr{R} \rightarrow A^{-1 / 2} \gamma^{3 / 2} \mathscr{R}$ we get $A=1$ and $\gamma=1$ in (1) above. For simplicity we will henceforth assume $A=1$ and $\gamma=1$.

If $K=1$ we get the TFW-theory for an atom. We will usually choose $\mathscr{R}_{1}=0$ in this case. For molecules we will denote the total nuclear charge by $Z=\sum_{j=1}^{K} z_{j}$.

In the TFW-theory we define the energy of a molecule with $N$ electrons ( $N$ not necessarily an integer), to be

$$
E(\underline{z} ; N)=\inf \left\{\mathscr{E}(\psi ; \underline{z} ; \mathscr{R}) \mid \psi \in G, \int_{\mathbb{R}^{3}} \psi^{2} d x \leqq N\right\} .
$$

In the present work we are not interested in the dependence on the nuclear coordinates so we do not write it explicitly.

It is known that there exists $N_{c}(z)>Z$ such that the variational problem (4) has a unique minimizer $\psi(x ; \underline{z} ; N) \geqq 0$ with $\int \psi^{2}=N$, if and only if $N \leqq N_{c}(z)$. We interpret

$$
Q_{c}(z)=N_{c}(z)-Z>0
$$

as the maximal (negative) ionization the molecule can achieve, i.e. the maximal "number" of extra electrons a neutral molecule can bind.

The function

$$
\varrho(x ; \underline{z} ; N)=\psi(x ; \underline{z} ; N)^{2}
$$

represents the electron density for the molecule. For $N \leqq N_{c}(z)$ the unique minimizer $\psi(\cdot ; z ; N)$ of (4) is the unique positive function $\psi \in G$ with $\int \psi^{2}=N$ satisfying the TFW-equation (The Euler-Lagrange equation for (4))

$$
-\Delta \psi+\left(\psi^{4 / 3}-\sum_{j=1}^{K} z_{j}\left|x-\mathscr{R}_{j}\right|^{-1}+\psi^{2} *|x|^{-1}\right) \psi=-\mu \psi,
$$

for a unique Lagrange multiplier $\mu=\mu(\underline{z} ; N)$. Here $*$ denotes convolution. Since $-\mu=\frac{\partial E}{\partial N}$, we call $-\mu(z ; N)$ the Chemical Potential. Since $E$ is a convex function of $N, \mu$ is decreasing in $N$ and there is a one to one correspondence between $\mu$ and $N$. 
When $N=N_{c}(\underline{z}), \psi(x ; \underline{z}) \equiv \psi\left(x ; \underline{z} ; N_{c}\right)$ is the total minimizer for $\mathscr{E}$ on the set $G$. In this case $\mu\left(z ; N_{c}\right)=0$. This case will also be referred to as the maximally ionized molecule.

We define the Potential Function,

$$
\varphi(x ; \underline{z} ; N)=\sum_{j=1}^{K} z_{j}\left|x-\mathscr{R}_{j}\right|^{-1}-\psi(\cdot ; \underline{z} ; N)^{2} *|x|^{-1} .
$$

In the atomic case the functions $\psi$ and $\varphi$ are radially symmetric. In the special case of the maximally ionized atom there exists a unique radius $R_{Z}>0$ such that $\partial_{r} \varphi\left(R_{Z}\right)=0$ or equivalently

$$
\int_{|x| \leqq R_{Z}} \varrho_{Z}(x) d^{3} x=Z,
$$

i.e. the total charge inside the ball of radius $R_{Z}$, counting both the electrons and the nucleus is zero. We call $R_{Z}$ the Radius of Neutrality for the atom.

Our main interest here is the behavior of the TFW model in the limit as some of the nuclear charges go to infinity. We could of course let all the nuclear charges go to infinity but we will consider the more general case.

If $1 \leqq L \leqq K$ we will consider $z_{j} \rightarrow \infty$, for $j=1, \ldots, L$ while $z_{j} j=L+1, \ldots, K$ are fixed. We denote $z^{\prime}=\left(z_{1}, \ldots, z_{L}\right)$ and $\underline{z}^{\prime \prime}=\left(z_{L+1}, \ldots, z_{K}\right)$. We will write $z^{\prime} \rightarrow \infty$ meaning $z_{j} \rightarrow \infty$ for $j=1, \ldots, L$. In Benguria and Lieb [2] it was proved that $Q_{c}(z)$ is bounded by a constant uniformly in $z$. Here we will prove that $Q_{c}$ actually converges as $\underline{z}^{\prime} \rightarrow \infty$. This was conjectured in [2]. Furthermore we will prove that the electron density for the maximally ionized molecule converges away from $\mathscr{R}_{1}, \ldots, \mathscr{R}_{L}$. At these points the limit density will have singularities that are not in $L^{1}$, this reflects the fact that the "limit molecule" has an infinite number of electrons, that clump together near the big nuclei. It will also be possible for us to give a surprisingly precise description of the order of the singularities (see Theorem 6 below) and thereby uniquely characterize the limit density. We will not restrict our attention to the maximally ionized case, but in general specify how $N$ tends to infinity with $z^{\prime}$. There are two different cases. We either specify that $\mu(\underline{z} ; N)$ or $N-Z$ should be fixed as $\underline{z}^{\prime}$ goes to infinity. The maximally ionized case corresponds to fixing $\mu=0$. The main results are summarized in the following theorems.

Theorem 1. If $\mu \geqq 0$ there exist functions $\psi_{\mu}\left(\cdot ; \underline{z}^{\prime \prime}\right)>0, \varphi_{\mu}\left(\cdot ; \underline{z}^{\prime \prime}\right)$ and an excess charge $Q\left(\underline{z}^{\prime \prime} ; \mu\right) \in \mathbb{R}$ such that if we fix $\mu(\underline{z} ; N)=\mu$ then

$$
\lim _{z^{\prime} \rightarrow \infty} \psi(x ; \underline{z} ; N)=\psi_{\mu}\left(x ; \underline{z}^{\prime \prime}\right),
$$

uniformly on the complement of any neighborhood of $\left\{\mathscr{R}_{1}, \ldots, \mathscr{R}_{L}\right\}$,

$$
\lim _{z^{\prime} \rightarrow \infty} \varphi(x ; z ; N)=\varphi_{\mu}\left(x ; z^{\prime \prime}\right),
$$

in $L_{\text {ioc }}^{p}\left(\mathbb{R}^{3} \backslash\left\{\mathscr{R}_{1}, \ldots, \mathscr{R}_{L}\right\}\right)$, all $p<3$, and uniformly on the complement of any neighborhood of $\left\{\mathscr{R}_{1}, \ldots, \mathscr{R}_{K}\right\}$,

$$
\lim _{\underline{z}^{\prime} \rightarrow \infty}(N-Z)=Q\left(\underline{z}^{\prime \prime} ; \mu\right) .
$$


Theorem 2. There exists $Q_{\infty}\left(\underline{z}^{\prime \prime}\right)>0$ such that

$$
\lim _{\underline{z}^{\prime} \rightarrow \infty} Q_{c}(\underline{z})=Q_{\infty}\left(\underline{z}^{\prime \prime}\right) .
$$

Corollary 3. In the case of the maximally ionized atom there exists $R_{\infty} \in(0, \infty)$ such that

$$
\lim _{Z \rightarrow \infty} R_{Z}=R_{\infty} .
$$

Remark. If $\mu(\underline{z} ; N)=0$ then $N=N_{c}(\underline{z})$. The existence of the limit in (13) therefore follows from (12) and $Q\left(z^{\prime \prime} ; \mu=0\right)=Q_{\infty}\left(z^{\prime \prime}\right)$. The only part of Theorem 2 which does not follow from Theorem 1 is the statement that $Q_{\infty}\left(z^{\prime \prime}\right)>0$. A priori it is not clear that the "limit molecule" can have a negative ionization.

Theorem 4. If $Q \in\left(-\infty, Q_{\infty}\left(\underline{z}^{\prime \prime}\right)\right)$ then $Q<Q_{c}(z)$ for $\underline{z}^{\prime}$ large enough. There exists a chemical potential $\mu\left(z^{\prime \prime} ; Q\right) \in \mathbb{R}_{+}$such that if we fix $N-Z=Q$, then

$$
\lim _{\underline{z}^{\prime} \rightarrow \infty} \mu(z ; N)=\mu\left(\underline{z}^{\prime \prime} ; Q\right) \text {. }
$$

The function $\mu\left(z^{\prime \prime} ; \cdot\right)$ is the inverse of $Q\left(z^{\prime \prime} ; \cdot\right)$. Again fixing $N-Z=Q$ we find with the notation of Theorem 1 and writing $\mu=\mu\left(z^{\prime \prime} ; Q\right)$,

$$
\lim _{z^{\prime} \rightarrow \infty} \psi(x ; \underline{z} ; N)=\psi_{\mu}\left(x ; \underline{z}^{\prime \prime}\right) \text {, and } \lim _{\underline{z}^{\prime} \rightarrow \infty} \varphi(x ; \underline{z} ; N)=\varphi_{\mu}\left(x ; \underline{z}^{\prime \prime}\right) \text {, }
$$

in the same sense as in Theorem 1.

Remark. $Q=0$ corresponds to neutral molecules. Hence $-\mu\left(z^{\prime \prime} ; 0\right)$ is the limit of the chemical potentials for very large neutral molecules.

Corollary 5. With the assumptions of Theorem 4 we get the convergence of the ionization energy

$$
\lim _{\underline{z}^{\prime} \rightarrow \infty}(E(\underline{z} ; Z)-E(\underline{z} ; Z+Q))=\mathscr{E}\left(\underline{z}^{\prime \prime} ; Q\right),
$$

where $\mathscr{E}\left(\underline{z}^{\prime \prime} ; Q\right) \in\left(-\infty, \mathscr{E}_{\max }\left(\underline{z}^{\prime \prime}\right)\right), \mathscr{E}_{\max }=\mathscr{E}\left(\underline{\underline{z}}^{\prime \prime} ; Q_{\infty}\right)$.

Proof. This is an easy consequence of the dominated convergence theorem and (15), since $-\mu=\frac{\partial E}{\partial N}$. Indeed we get $\mathscr{E}\left(\underline{z}^{\prime \prime} ; Q\right)=\int_{0}^{Q} \mu\left(\underline{\underline{z}}^{\prime \prime} ; Q^{\prime}\right) d Q^{\prime}$.

The next theorem gives the asymptotic expansions near each $\mathscr{R}_{j}, j=1, \ldots, L$. The surprising conclusion of this theorem is that even if the $\mathscr{R}_{j}$ 's are distributed in a highly non-symmetric way, both $\varphi$ and $\psi$ will be spherically symmetric to very high order near $\mathscr{R}_{j}, j=1, \ldots, L$.

Theorem 6. (a) Asymptotically near each $\mathscr{R}_{j}, j=1, \ldots, L$,

$$
\begin{aligned}
\psi_{\mu}\left(x+\mathscr{R}_{j} ; \underline{z}^{\prime \prime}\right)= & 3 \sqrt{3} \pi^{-3 / 2}|x|^{-3}-\frac{3 \sqrt{3}}{16} \pi^{1 / 2}|x|^{-1}-\frac{3 \sqrt{3}}{512} \pi^{5 / 2}|x| \\
& -\frac{323 \sqrt{3}}{73728} \pi^{9 / 2}|x|^{3}+O\left(|x|^{\sigma+1}\right),
\end{aligned}
$$


and

$$
\varphi_{\mu}\left(x+\mathscr{R}_{j} ; z^{\prime \prime}\right)-\mu=9 \pi^{-2}|x|^{-4}-\frac{27}{4}|x|^{-2}-\frac{25}{64} \pi^{2}-\frac{37}{768} \pi^{4}|x|^{2}+O\left(|x|^{\sigma}\right),
$$

where $\sigma=-1 / 2+\sqrt{73} / 2 \approx 3.8$.

(b) The asymptotic form at infinity. If $\mu=0$

$$
\lim _{|x| \rightarrow \infty}|x|^{-1 / 2} \log \psi_{0}\left(x ; \underline{z}^{\prime \prime}\right)=-2 \sqrt{Q_{\infty}\left(\underline{z}^{\prime \prime}\right)} \text {. }
$$

If $\mu \neq 0$

$$
\lim _{|x| \rightarrow \infty}|x|^{-1} \log \psi_{\mu}\left(x ; \underline{z}^{\prime \prime}\right)=-\mu
$$

For all $\mu \geqq 0$

$$
\lim _{|x| \rightarrow \infty}|x| \varphi_{\mu}\left(x ; \underline{z}^{\prime \prime}\right)=-Q\left(\underline{z}^{\prime \prime} ; \mu\right) .
$$

Remark. The highest order terms for the asymptotic expansions of $\varphi$ and $\psi$ in (a) are the same as in the TF-theory. The power $\sigma$ also appears here (see Veron [20] or Sommerfeld [18]). The asymptotic forms (20)-(22) hold, with the obvious changes, for finite $\underline{z}$ also. These properties for finite $\underline{z}$, especially that $\psi$ is exponentially decaying at infinity, and that $\varphi<0$ for large $|x|$ if $N>Z$, will be used throughout this work.

As an important step in the proofs of Theorems $1-4$ we give a unique characterization of the functions $\varphi_{\mu}$ and $\psi_{\mu}$. This characterization which is interesting in itself uses the following renormalization procedure. Define the Renormalized Electron Density,

$$
\varrho_{\mu}^{(2)}\left(x ; \underline{z}^{\prime \prime}\right)=\psi_{\mu}\left(x ; \underline{z}^{\prime \prime}\right)^{2}-\sum_{j=1}^{L}\left(d_{1}\left|x-\mathscr{R}_{j}\right|^{-6}+d_{2}\left|x-\mathscr{R}_{j}\right|^{-4}\right),
$$

where $d_{1}=27 \pi^{-3}$ and $d_{2}=-(27 / 8) \pi^{-1}$ are computed from (18). Then

Theorem 7. $\psi_{\mu}$ is the unique positive function on $\mathbb{R}^{3}$ such that $\varrho_{\mu}^{(2)} \in C_{b}\left(\mathbb{R}^{3}\right) \cap L^{1}\left(\mathbb{R}^{3}\right)$ $\left(C_{b}=\right.$ continuous and bounded), and which satisfies the Renormalized TFWequation

$$
\begin{aligned}
& -\Delta \psi_{\mu}+\left\{\psi_{\mu}^{4 / 3}-\sum_{j=1}^{L}\left(a_{1}\left|x-\mathscr{R}_{j}\right|^{-4}+a_{2}\left|x-\mathscr{R}_{j}\right|^{-2}\right)\right. \\
& \left.-\sum_{j=L+1}^{K} z_{j}\left|x-\mathscr{R}_{j}\right|^{-1}+\varrho_{\mu}^{(2)} *|x|^{-1}+\mu\right\} \psi_{\mu}=0,
\end{aligned}
$$

on $\mathbb{R}^{3} \backslash\left\{\mathscr{R}_{1}, \ldots, \mathscr{R}_{L}\right\}$, where $a_{1}=9 \pi^{-2}$ and $a_{2}=-27 / 4$. $\varphi_{\mu}$ is given $b y$

$$
\varphi_{\mu}(x)=\sum_{j=1}^{L}\left(a_{1}\left|x-\mathscr{R}_{j}\right|^{-4}+a_{2}\left|x-\mathscr{R}_{j}\right|^{-2}\right)+\sum_{j=L+1}^{K} z_{j}\left|x-\mathscr{R}_{j}\right|^{-1}-\varrho_{\mu}^{(2)} *|x|^{-1} \text {. }
$$

$\varrho_{\mu}^{(2)}$ satisfies

$$
\int \varrho_{\mu}^{(2)}(x) d^{3} x=Q\left(\underline{z}^{\prime \prime} ; \mu\right)+\sum_{j=\boldsymbol{L}+1}^{K} z_{j} \equiv Q+Z^{\prime \prime} .
$$

Remark. Equation (26) illustrates the role of $\varrho_{\mu}^{(2)}$ as a renormalized density. In Theorem 37 below we give a different characterization of $\varphi_{\mu}$ and $\psi_{\mu}$.

The proofs of the theorems presented here will be given in Sect. 8 . We will conclude this introduction by describing the general ideas. 
Theorems 1-4 state that in the TFW-theory the configuration of the outer electrons becomes more or less independent of the nuclear charges when these charges are very large. This implies on the one hand that molecules do not become very big and on the other that they do not collapse as the nuclear charges go to infinity. If electrons were treated as bosons, i.e., $\gamma=0$ in (1) molecules would collapse as $z_{1}, \ldots, z_{K}$ become very large, i.e. the electrons would all sit on top of the nuclei.

The idea in the proof of the main theorems is to prove first that molecules remain bounded. That is to give upper bounds to $\psi$ uniformly in $\underline{z}$. This is done in Sect. 3. Next we prove in Sect. 4 that molecules do not collapse, i.e. uniform lower bounds. In Sect. 5 we prove that any sequence of $z$ 's and $N$ 's has a subsequence such that the convergences in Theorems 1-4 hold. To prove the theorems we have to prove that all subsequences have the same limit. To conclude this uniqueness property we first prove in Sect. 6 that the limits $\varphi$ and $\psi$ of any subsequence satisfy the asymptotic expansions in Theorem 6. In Sect. 7 we use the asymptotic expansions to derive the renormalized TFW-equation. We finish the proof of the main theorems by proving that the solution to this equation is unique.

In Benguria and Lieb [2] a series of estimates independent of $\underline{z}$ were derived for $\varphi$ and $\psi$. These estimates subsequently yielded an upper bound to $Q_{c}(z)$ independent of $z$. In the present work we will rely heavily on these estimates. For the sake of completeness and since we improve some of the results in [2] we summarize these estimates in Sect. 2.

In [13] Rother gets upper and lower bounds on $\varphi, \psi$, and $Q_{c}$. Unfortunately these bounds are not uniform in $\underline{z}$.

In [17] the atomic case was studied numerically. It was found that in terms of real units with the choice of $A$ that reproduces the Scott correction, i.e., $A=0.1859$ and $\gamma=\left(3 \pi^{2}\right)^{2 / 3}$,

$$
Q_{\infty}=0.031 e \text { and } R_{\infty}=18.1 a_{0},
$$

where $e$ is the electron charge and $a_{0}$ is the Bohr radius. This might seem like a very bad value for $Q_{\infty}$ compared to the expected physical value $Q_{\infty}=1$. That this is

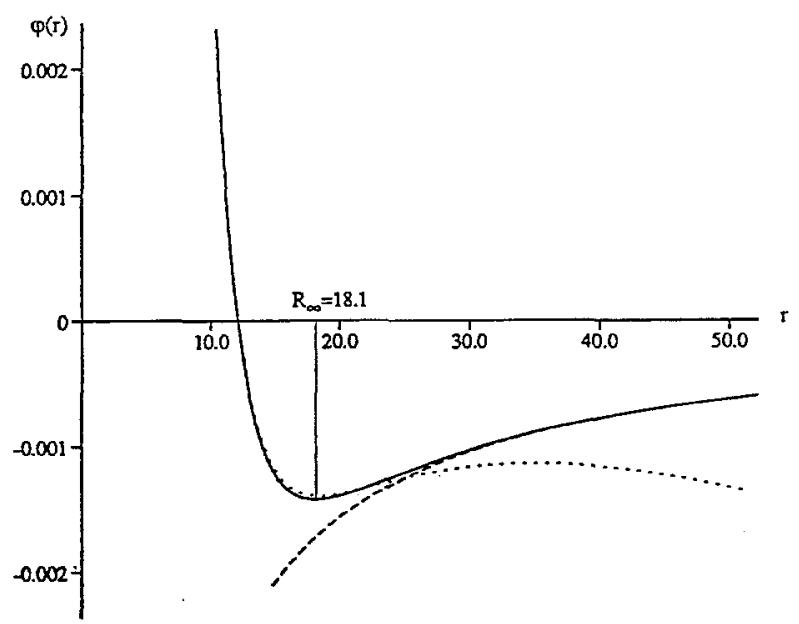

Fig. 1. $\varphi_{\infty}$ (solid curve) and its asymptotic forms at 0 (dotted curve) and at $\infty$ (dashed curve). The unit for $r$ is $a_{0}$, the unit for $\varphi$ is $a_{0}^{-1}$ 
really not too bad can be explained from the fact that if we restrict ourselves to integer values for $N$ and ask when is the energy smallest we find

$$
E(N=Z, Z)>E(N=Z+1, Z)=E(N=Z+2, Z)\left(=E\left(N=Z+Q_{c}, Z\right)\right) .
$$

We would then conclude that it is possible to ionize an atom with exactly one extra electron.

Alternatively we can take into account the fact that an electron is not interacting with itself by introducing the Fermi-Amaldi correction (see also [2]), i.e., replace $D\left(\psi^{2}, \psi^{2}\right)$ in $(1)$ by $(1-1 / N) D\left(\psi^{2}, \psi^{2}\right)$. The effect of this is to multiply $A$, $\gamma$, and $Z$ by the factor $N /(N-1)$. The critical number of electrons is then 1.03 .

In the Thomas-Fermi-Dirac-von Weizsäcker (TFDW) model this effect is taken into account by introducing an exchange term in the energy functional. A numerical analysis has been made for a modified TFDW model in [19] (with $A=0.2$ ). For the critical charge the result is $Q_{c}=0.3$ (Dreizler [4]).

The graph for $\varphi_{\infty}$ for the universal (infinite) maximally ionized TFW-atom is shown in Fig. 1. The unit for $r$ is $a_{0}$, the unit for $\varphi$ is $a_{0}^{-1}$. The figure also shows the asymptotic forms given in Theorem 6 . It is worth noticing how accurate the asymptotic curve agrees with the numerical solution even for fairly large values of $r\left(r \approx 15-20 a_{0}\right)$. More detailed results are given in [17].

We finally mention that it is an interesting open problem to prove that $Z \mapsto Q_{c}(Z)$ is an increasing function.

\section{The Estimates of Benguria and Lieb}

With $\varphi$ given in (8) we can write the TFW-equation as

$$
-\Delta \psi+\left(\psi^{4 / 3}-\varphi+\mu\right) \psi=0
$$

Notice that we also have

$$
\Delta \varphi(x)=4 \pi\left(\psi^{2}(x)-\sum_{j=1}^{K} z_{j} \delta\left(x-\mathscr{R}_{j}\right)\right) .
$$

We will call (27)-(28) the TFW-system.

Proposition 8 (Benguria and Lieb). For all $N$, and $\underline{z}$

$$
\lambda \psi(x ; \underline{z} ; N)^{4 / 3} \leqq \varphi(x ; \underline{z} ; N)+[C(\lambda)-\mu(\underline{z} ; N)]_{+}
$$

for all $\lambda \in(0,1)$ and all $x \in \mathbb{R}^{3}$, where $C(\lambda)=(9 / 4) \pi^{2} \lambda^{-2}(1-\lambda)^{-1}$.

We use the notation $[a]_{+}=\max \{a, 0\}$ for $a \in \mathbb{R}$.

Proof. Let $u(x)=\psi(x)^{4 / 3}$. Then from (27)

$$
-\Delta u+(4 / 3)(u-\varphi+\mu) u \leqq 0 .
$$

Let $v(x)=\lambda u(x)-\varphi(x)-[C(\lambda)-\mu]_{+}$. From (28) and (30) we obtain

$$
\Delta v(x) \geqq(4 / 3) \lambda(u-\varphi+\mu) u-4 \pi u^{3 / 2}, \quad x \neq \mathscr{R}_{j}, \text { all } j .
$$

Let $S=\{x \mid v(x)>0\}$. Our aim is to prove that $S=\emptyset$. Clearly $\mathscr{R}_{j} \notin S$ for all $j$. Thus on $S$

$$
\begin{aligned}
\Delta v(x) & \geqq(4 / 3) \lambda u\left(u-\lambda u+[C(\lambda)-\mu]_{+}+\mu\right)-4 \pi u^{3 / 2} \\
& \geqq u\left[(4 / 3) \lambda(1-\lambda) u-4 \pi u^{1 / 2}+(4 / 3) \lambda C(\lambda)\right] \geqq 0
\end{aligned}
$$


with our choice of $C(\lambda)$. Since $u(x), \varphi(x) \rightarrow 0$ as $x \rightarrow \infty, v(x) \rightarrow-[C(\lambda)-\mu]_{+} \leqq 0$. If $S$ is bounded $v=0$ on $\partial S$ and it follows that $S=\emptyset$, since $v$ is subharmonic on $S$. If $S$ is unbounded $v(x) \rightarrow 0$ as $x \rightarrow \infty$ in $S$ and we conclude again that $S=\emptyset$.

Corollary 9. For the chemical potential of a neutral system we get

$$
-\mu(z ; N=Z) \geqq-3^{5} 2^{-4} \pi^{2}
$$

Proof. $C(\lambda)$ takes its minimal value $3^{5} 2^{-4} \pi^{2}$ at $\lambda=2 / 3$. If $\mu>3^{5} 2^{-4} \pi^{2}$ we get from Proposition 8 that $\varphi(x) \geqq \lambda \psi(x)>0$ for all $x$. Hence we must have $N \leqq Z$. Since $\mu$ is continuous in $N$ for fixed $z$ we conclude that $N<Z$.

The bound (33) is an improvement of the bound given in [2, Theorem 7] which replaces $3^{5} 2^{-4} \pi^{2}(\approx 150)$ by $27 \pi^{2}(\approx 266)$.

Later on we will need to extend the bound in (33) to $Q=N-Z<0$. Such a bound is more complicated and we have to wait until Sect. 5 to give the proof [see Proposition 29 proof of case $(Q)]$.

In the next lemma which is also in [2] we derive an estimate which in some sense is converse to Proposition 8 . Let $e_{R}(x)$ be the normalized ground state of the Dirichlet Laplacian on the ball of radius $R$ centered at the origin, i.e., $e_{R}(x)$ $=(2 \pi R)^{-1 / 2}|x|^{-1} \sin \left(\pi R^{-1}|x|\right)$ for $|x| \leqq R$ and $e_{R}(x)=0$ otherwise. Then $\int\left|\nabla e_{R}\right|^{2} d x$ $=\pi^{2} R^{-2}$. Define $g_{R}(x)=e_{R}(x)^{2}$.

Lemma 10. Let $\Omega \subseteq \mathbb{R}^{3}$ be any open set. If $0<\psi \in H_{0}^{2}(\Omega)$ satisfies $E q$. (27) on $\Omega$ with $\varphi \in L^{2}(\Omega)+L^{\infty}(\Omega)$, then for all $x \in \Omega$ with $\operatorname{dist}(x, \partial \Omega)>R$,

$$
g_{R} * \varphi(x) \leqq\left(g_{R} * \psi^{4 / 3}\right)(x)+\mu+\pi^{2} R^{-2} .
$$

Furthermore if $\Omega$ does not contain any nuclei we conclude

$$
\varphi(x) \leqq g_{R} * \varphi(x) .
$$

Proof. Since $0<\psi, \psi$ is the ground state for $H=-\Delta_{D}+\left(\psi^{4 / 3}-\varphi\right)$. Here $\Delta_{D}$ denotes the Dirichlet Laplacian on $\Omega$. Thus for all $\chi \in H_{0}^{1}(\Omega)$,

$$
\int_{\Omega}|\nabla \chi|^{2}+\int_{\Omega}\left(\psi^{4 / 3}-\varphi\right)|\chi|^{2} d x \geqq-\mu \int_{\Omega}|\chi|^{2} d x .
$$

Using this inequality on $\chi=e_{R}(x-\cdot)$, where dist $(x, \partial \Omega)>R$ gives (34). If $\varphi$ satisfies (28), $\varphi$ is subharmonic on $\Omega . g_{R}$ is spherically symmetric, positive and of total mass one. This implies (35).

In $[2$, Lemma 10] was used to prove an upper bound to $\varphi(x)$ independent of the $z_{j}$. Unfortunately this upper bound is not completely satisfactory near the nuclei. Here we will give an improved bound which is optimal to leading order near the nuclei. To do this we first notice (see also [3]) that if $t$ solves $t(t+1)=18$, then for all $k \in \mathbb{R}$,

$$
\omega \equiv 9 \pi^{-2}|x|^{-4}+k|x|^{t},
$$

satisfies

$$
\Delta \omega(x) \leqq 4 \pi \omega(x)^{3 / 2} \text { for }|x| \neq 0
$$


on the set where $\omega \geqq 0$. We denote the positive root of $t(t+1)=18$ by $\sigma$ and the negative root by $-\tau$. Then $\sigma=-\frac{1}{2}+\frac{\sqrt{73}}{2}$ is the exponent that appears in Theorem $6, \tau=\frac{1}{2}+\frac{\sqrt{73}}{2}>4$ is used in the following

Lemma 11. Assume $\hat{\varphi}$ is a positive function on the set $\{x|| x \mid>R\}, \hat{\varphi}$ is bounded, goes to zero at infinity and satisfies $\Delta \hat{\varphi}=4 \pi \hat{\varphi}^{3 / 2}$ on $\{x|| x \mid>R\}$. Then

$$
\hat{\varphi}(x) \leqq \chi(R /|x|)|x|^{-4} \text { on }\{x|| x \mid>R\} .
$$

Where $\chi:[0,1) \rightarrow \mathbb{R}_{+}$is defined by

$$
\chi(\alpha)=\left\{\begin{array}{lll}
9 \pi^{-2}+C \alpha^{\tau-4} & \text { if } & 0 \leqq \alpha \leqq \alpha_{0} \\
25 \pi^{-2}(1-\alpha)^{-4} & \text { if } & \alpha_{0}<\alpha<1
\end{array}\right.
$$

Here $\left(C, \alpha_{0}\right)$ is the unique pair which makes $\chi \in C^{1}([0,1))$.

Remark. We have $C \approx 167.6 \pi^{-2}$ and $\alpha_{0} \approx 1 / 7$.

Proof. We first notice that

$$
\hat{\varphi}(x) \leqq 25 \pi^{-2}(|x|-R)^{-4} \text { for }|x|>R .
$$

This follows easily from a comparison argument using that $f(x)$ $\equiv 25 \pi^{-2}(|x|-R)^{-4}$ satisfies $\Delta f \leqq 4 \pi f^{3 / 2}$ and $f(x) \rightarrow \infty$ as $|x| \rightarrow R$.

From the choice of $\left(C, \alpha_{0}\right)$ we find that for $|x|=\alpha_{0}^{-1} R, f(x)=\omega(x)$, where $\omega$ is defined as in (37) with $k=C R^{\tau-4}$. By the very same comparison argument as for $f$ we conclude that $\hat{\varphi}(x) \leqq \omega(x)$ for $|x| \geqq \alpha_{0}^{-1} R$. This together with (41) gives (39).

We can now give the improved version of the bound of Benguria and Lieb, by simply copying their proof.

Proposition 12 (Benguria and Lieb). For all $\alpha \in(0,1)$ and all $x$,

$$
\varphi(x ; z ; N)-\mu(z ; N) \leqq \sum_{j=1}^{K}\left(\chi(\alpha)\left|x-\mathscr{R}_{j}\right|^{-4}+\pi^{2} \alpha^{-2}\left|x-\mathscr{R}_{j}\right|^{-2}\right),
$$

where $\chi(\alpha)$ is given in $(40)$.

Proof. Given $R>0$. For all $x \in \mathbb{R}^{3}$ we get from Lemma 10 ,

$$
g_{R} * \varphi(x)-\mu \leqq\left(g_{R} * \psi^{4 / 3}\right)(x)+\pi^{2} R^{-2} .
$$

Using Hölder's inequality and the fact $\int g_{R}=1$ we obtain

$$
\widetilde{\varphi}(x) \equiv g_{R} * \varphi(x)-\mu-\pi^{2} R^{-2} \leqq\left(g_{R} * \varrho\right)^{2 / 3}(x) .
$$

On the set $\left\{x|| x-\mathscr{R}_{j} \mid>R\right.$, all $\left.j\right\}$, we get using (35) that

$$
\varphi(x)-\mu \leqq \widetilde{\varphi}(x)+\pi^{2} R^{-2} \text {. }
$$

Convoluting on both sides of $(4 \pi)^{-1} \Delta \varphi=-\sum_{j} z_{j} \delta\left(x-\mathscr{R}_{j}\right)+\varrho$ we find

$$
(4 \pi)^{-1} \Delta \tilde{\varphi} \geqq-\sum_{j=1}^{K} z_{j} g_{R}\left(x-\mathscr{R}_{j}\right)+[\tilde{\varphi}]_{+}^{3 / 2} .
$$


Since $\tilde{\varphi}$ is continuous and converges to $-\mu-\pi^{2} R^{-2}$ at infinity it is easy to compare it with the corresponding TF-potential. That is the positive function $\hat{\varphi}$ which satisfies

$$
(4 \pi)^{-1} \Delta \hat{\varphi}=-\sum_{j=1}^{K} z_{j} g_{R}\left(x-\mathscr{R}_{j}\right)+\hat{\varphi}^{3 / 2}
$$

We find

$$
\widetilde{\varphi}(x) \leqq \hat{\varphi}(x) .
$$

It is known (Lieb and Simon [11, Theorem V.12] or Lieb [10, Corollary 3.6]) that $\hat{\varphi}(x) \leqq \sum_{j=1}^{K} \hat{\varphi}_{j}(x)$, where $\hat{\varphi}_{j}$ is the TF-solution for an atom with nuclear charge density $z_{j} g_{R}\left(x-\mathscr{R}_{j}\right)$. Then $\hat{\varphi}_{j}$ satisfies the assumptions in Lemma 11, and

$$
\hat{\varphi}_{j}(x) \leqq \chi\left(\frac{R}{\left|x-\mathscr{R}_{j}\right|}\right)\left|x-\mathscr{R}_{j}\right|^{-4} \quad \text { on } \quad\left\{x|| x-\mathscr{R}_{j} \mid \geqq R\right\} .
$$

For all $x$ satisfying $\left|x-\mathscr{R}_{j}\right|>R$, all $j$, we hence get

$$
\varphi(x)-\mu \leqq \sum_{j=1}^{K} \chi\left(\frac{R}{\left|x-\mathscr{R}_{j}\right|}\right)\left|x-\mathscr{R}_{j}\right|^{-4}+\pi^{2} R^{-2} .
$$

For any $x \neq \mathscr{R}_{j}$, all $j$, we now choose $R=\alpha \min _{j}\left|x-\mathscr{R}_{j}\right|$. Then

$$
R^{-2} \leqq \sum_{j} \alpha^{-2}\left|x-\mathscr{R}_{j}\right|^{-2}
$$

and since $\chi$ is an increasing function we obtain (42).

Remark. In [2] the bound (42) was proved with $\chi(\alpha)=25 \pi^{-2}(1-\alpha)^{-4}$. The $\chi$ given here satisfies $\lim _{\alpha \rightarrow \infty} \chi(\alpha)=9 \pi^{-2}$. This limit is optimal (compare Theorem 6 and the remark after Lemma 21).

Remark. In Proposition $12 \varphi$ is bounded in terms of $\mu(z ; N)$. It is thus important as mentioned earlier to prove that $\mu$ is bounded if $Q=N-Z$ is fixed (see Proposition 29).

\section{Upper Bounds}

In this section we will prove that the electrons stay in a bounded region as $\underline{z} \rightarrow \infty$. More precisely we will prove that the $L^{2}$-norm of $\psi$ on the set $\{x|| x \mid \geqq r\}$ goes to zero uniformly in $z$ when $r \rightarrow \infty$. From Propositions 8 and 12 we only know that $\psi$ is uniformly bounded by a constant for large $|x|$. Our first step is

Lemma 13. For all $\alpha \in(0,1)$ and all $x$,

$$
\psi(x ; z ; N)^{4 / 3} \leqq\left(1+\frac{9 \alpha^{2}}{2 \pi^{2}}\right) \sum_{j=1}^{K}\left(\chi(\alpha)\left|x-\mathscr{R}_{j}\right|^{-4}+\pi^{2} \alpha^{-2}\left|x-\mathscr{R}_{j}\right|^{-2}\right),
$$

where $\chi$ is given in (40). 
Proof. Let $f_{j}(x)=\beta\left(\chi(\alpha)\left|x-\mathscr{R}_{j}\right|^{-4}+\pi^{2} \alpha^{-2}\left|x-\mathscr{R}_{j}\right|^{-2}\right)$, where $\beta>1$. Define $f(x)$ $=\sum f_{j}(x)$. Proposition 12 states that $\beta(\varphi-\mu) \leqq f$. Consider the set $S=\left\{x \mid \psi^{4 / 3}(x)>f(x)\right\}$. Then clearly $\mathscr{R}_{j} \notin S$, all $j$, and since $\psi$ decays exponentially at infinity, $S$ is open and bounded. On $S$ we have $\psi^{4 / 3}>\beta(\varphi-\mu)$. Hence the TFWequation (7) gives

$$
\Delta \psi^{4 / 3} \geqq(4 / 3)\left(\psi^{4 / 3}-(\varphi-\mu)\right) \psi^{4 / 3}>(4 / 3)\left(1-\beta^{-1}\right)\left(\psi^{4 / 3}\right)^{2} .
$$

On the other hand for $x \neq \mathscr{R}_{j}$,

$$
\Delta f_{j}=\beta\left(12 \chi(\alpha)\left|x-\mathscr{R}_{j}\right|^{-6}+2 \pi^{2} \alpha^{-2}\left|x-\mathscr{R}_{j}\right|^{-4}\right) \leqq 6\left(\pi^{2} \alpha^{-2} \beta\right)^{-1} f_{j}^{2} .
$$

Hence

$$
\Delta f \leqq 6\left(\pi^{2} \alpha^{-2} \beta\right)^{-1} \sum_{j=1}^{K} f_{j}^{2} \leqq 6\left(\pi^{2} \alpha^{-2} \beta\right)^{-1} f^{2}
$$

for $x \neq \mathscr{R}_{j}$. If we choose $6\left(\pi^{2} \alpha^{-2} \beta\right)^{-1}=4 / 3\left(1-\beta^{-1}\right)$, i.e., $\beta=1+(9 / 2) \pi^{-2} \alpha^{2}$, then $\psi^{4 / 3}-f$ is subharmonic on $S$. On $\partial S, \psi^{4 / 3}-f=0$, we conclude that $S=\emptyset$.

With this lemma we can now improve the upper bound $Q_{c} \leqq 270.74 K$ given in [2].

Proposition 14. $Q_{c}(z) \leqq 178.03 K$.

Proof. As realized in Benguria-Lieb [2] it is enough to consider the atomic case $K=1$. As in [2] we use the fact that if we define

$$
p(x)=\left(4 \pi \psi(x)^{2}+\varphi(x)^{2}\right)^{1 / 2},
$$

then $Q_{c} \leqq|x| p(x)$ for all $x \neq 0$. We choose to estimate $|x| p(x)$ at $|x|=0.9086$. If at this point $\varphi(x)>0$ we use Proposition 12 to estimate $\varphi$ and Lemma 13 to estimate $\psi(\mu=0)$. In both cases we take $\alpha=0.4424$. We get $Q_{c} \leqq 141.03$. If $\varphi(x)<0$, we use the estimate in [2] (p. 1052 formula (44)): $Q_{c} \leqq 178.03$.

Remark. Compared with the numerical value $Q_{\infty}=11.54$ (with $A=1$ and $\gamma=1$, see [17]) this is an order of magnitude wrong.

Unfortunately the bound on $\psi$ given in Lemma 13 is not in $L^{2}$. To get a better estimate we will consider the problem of finding $\psi$ outside the ball $\{x|| x \mid>r\}$. The origin $x=0$ is arbitrary but should be thought of as being somewhere in the center of the molecule, $r$ should then be so large that $\left|\mathscr{R}_{j}\right|<r$ for all $j$.

For the outside problem of finding $\psi$ on $\{x|| x \mid>r\}$ the function

$$
\tilde{\varphi}_{r}(x) \equiv \sum_{j=1}^{K} z_{j}\left|x-\mathscr{R}_{j}\right|^{-1}-j_{|y| \leqq r} \varrho(y)|x-y|^{-1} d y-\mu,
$$

will be considered as known.

Lemma 15. There exists a constant $C>0$, such that for $r>\max _{j}\left|\mathscr{R}_{j}\right|+1$,

$$
\tilde{\varphi}_{r}(x) \leqq C r^{-1 / 2}|x|^{-1}
$$

on the set $\{x|| x \mid \geqq r\}$. 
Proof. Let $\delta=\left(\max \left|\mathscr{R}_{j}\right|+1\right)^{1 / 2}$ then $r^{\prime} \equiv \delta r^{1 / 2}$ clearly satisfies $\max \left|\mathscr{R}_{j}\right|+1<r^{\prime} \leqq r$.

On the set $\left\{|x|>r^{\prime}\right\}, \tilde{\varphi}_{r}$ is subharmonic. An easy comparison argument shows that for all $|x| \geqq r^{\prime}$,

$$
\tilde{\varphi}_{\boldsymbol{r}}(x) \leqq \sup _{|y|=\boldsymbol{r}^{\prime}}\left(r^{\prime} \tilde{\varphi}_{r}(y)\right)|x|^{-1}
$$

Now the definition (46) implies that

$$
\sup _{|y|=\boldsymbol{r}^{\prime}} \tilde{\varphi}_{\boldsymbol{r}}(y) \leqq \sup _{|y|=\boldsymbol{r}^{\prime}}(\varphi(y)-\mu)+\sup _{|\boldsymbol{y}|=\boldsymbol{r}^{\prime}} \int_{\left|y^{\prime}\right| \geqq \boldsymbol{r}} \varrho\left(y^{\prime}\right)\left|y-y^{\prime}\right|^{-1} d y^{\prime} .
$$

From Proposition 12, $\sup _{|y|=r^{\prime}}(\varphi(y)-\mu) \leqq \operatorname{const}\left(r^{\prime}\right)^{-2}$ and from Lemma 13,

$$
\begin{aligned}
\sup _{|y|=r^{\prime}} \int_{\left|y^{\prime}\right| \geqq r} \varrho\left(y^{\prime}\right)\left|y-y^{\prime}\right|^{-1} d y^{\prime} & \leqq \sup _{|y|=r^{\prime}} \int_{\left|y^{\prime}\right| \geqq r} \text { const }\left|y^{\prime}\right|^{-3}\left|y-y^{\prime}\right|^{-1} d y^{\prime} \\
& \leqq \text { const } \int_{\left|y^{\prime}\right| \geqq r}\left|y^{\prime}\right|^{-4} d y^{\prime} \leqq \text { const } r^{-1} .
\end{aligned}
$$

Hence

$$
\tilde{\varphi}_{r}(x) \leqq \operatorname{const}\left(r^{\prime-1}+r^{\prime} r^{-1}\right)|x|^{-1} \leqq C r^{-1 / 2}|x|^{-1}
$$

for all $x$ with $|x|>r^{\prime}$.

Lemma 15 states that on the set $\{|x|>r\}, \tilde{\varphi}_{r}$ is smaller than the potential coming from a central charge of size $\mathrm{Cr}^{-1 / 2}$. It is a well known fact (Lieb [10] or Lieb [9]) that the total number of electrons of an atom is of the order of the nuclear charge (for real atoms $N<2 Z+1$ ). Using the method of Lieb on the outside problem we can now prove that the amount of charge outside the ball of radius $r$ goes to zero uniformly in $z$ as $r$ goes to infinity. This idea is being used on the true quantum problem in [14].

Proposition 16. There exists a constant $M>0$ independent of $\underline{z}$ and $N$ such that for all $r>2\left(\max _{j}\left|\mathscr{R}_{j}\right|+1\right)$,

$$
\int_{|x| \geqq r} \psi(x ; \underline{z} ; N)^{2} d x \leqq M r^{-1 / 2} .
$$

Proof. Choose $\eta \in C^{\infty}\left(\mathbb{R}_{+}\right)$with supp $\eta \leqq[1, \infty), 0 \leqq \eta \leqq 1$ and $\eta(t)=1$ if $t \geqq 2$. Let $\eta_{r}: \mathbb{R}^{3} \rightarrow \mathbb{R}$ be defined by $\eta_{r}(x)=\eta(|x| / r)$. Then if $r>\max _{j}\left|\mathscr{R}_{j}\right|+1$,

$$
\begin{aligned}
\Delta\left(\eta_{r} \psi\right) & =\psi \Delta \eta_{\boldsymbol{r}}+2 \nabla \eta_{r} \nabla \psi+\left(\eta_{r} \psi\right)\left(\psi^{4 / 3}-\varphi+\mu\right) \\
& \geqq \psi \Delta \eta_{\boldsymbol{r}}+2 \nabla \eta_{r} \nabla \psi-\left(\eta_{r} \psi\right) \tilde{\varphi}_{\boldsymbol{r}}+\left(\eta_{\boldsymbol{r}} \psi\right) \int_{|y| \geqq r} \varrho(y)|x-y|^{-1} d y .
\end{aligned}
$$

We multiply this inequality by $|x| \eta_{r} \psi$, integrate over $\mathbb{R}^{3}$ and use (47),

$$
\begin{aligned}
\int|x| \eta_{r} \psi \Delta\left(\eta_{r} \psi\right) \geqq & \int|x| \eta_{r} \psi\left(\left(\Delta \eta_{r}\right) \psi+2 \nabla \eta_{r} \nabla \psi\right) d x-C r^{-1 / 2} \int\left(\eta_{r} \psi\right)^{2} d x \\
& +\int\left(\eta_{r} \psi\right)^{2}(y)|x-y|^{-1}|x|\left(\eta_{r} \psi\right)^{2}(x) d x d y .
\end{aligned}
$$


It is not difficult to see (Lieb [9]) that $\int|x|\left(\eta_{r} \psi\right) \Delta\left(\eta_{r} \psi\right) d x \leqq 0$. By symmetrization and using the triangle inequality

$$
\begin{gathered}
\int\left(\eta_{r} \psi\right)^{2}(y)|x-y|^{-1}|x|\left(\eta_{r} \psi\right)^{2}(x) d x d y \\
=(1 / 2) \int\left(\eta_{r} \psi\right)^{2}(y)|x-y|^{-1}(|x|+|y|)\left(\eta_{r} \psi\right)^{2}(x) d x d y \geqq(1 / 2)\left(\int\left(\eta_{r} \psi\right)^{2} d x\right)^{2} .
\end{gathered}
$$

Finally we estimate the "boundary" term, that is the first term in (49), using that for $|x| \geqq \max _{j}\left|\mathscr{R}_{j}\right|+1, \psi(x) \leqq$ const $|x|^{-3 / 2}$,

$$
\begin{aligned}
\left|\int\left(|x| \eta_{r} \Delta \eta_{r} \psi^{2}+|x| \eta_{r} \nabla \eta_{r} \nabla\left(\psi^{2}\right)\right) d x\right| & =\left|-\int\left(\eta_{\mathrm{r}} \frac{x}{|x|} \nabla\left(\eta_{r}\right) \psi^{2}+|x|\left(\nabla \eta_{r}\right)^{2} \psi^{2}\right) d x\right| \\
& \leqq \text { const } r^{-4} \int_{r}^{2 r} s^{2} d s=\text { constr }^{-1}
\end{aligned}
$$

Inserting the two above inequalities into (49) we arrive at

$$
0 \geqq- \text { constr } r^{-1}-C r^{-1 / 2} \int\left(\eta_{\boldsymbol{r}} \psi\right)^{2} d x+(1 / 2)\left(\int\left(\eta_{r} \psi\right)^{2} d x\right)^{2} .
$$

The right-hand side here is a quadratic expression in $\int\left(\eta_{r} \psi\right)^{2} d x$. We conclude that

$$
\int_{|x| \leqq 2 r} \psi(x)^{2} d x \leqq \int\left(\eta_{r} \psi\right)^{2} d x \leqq M r^{-1 / 2} .
$$

Later on (Theorem 28) we will prove a uniform (in z) exponential bound on $\psi$. The reason why we cannot prove this now is that we have to know that $Q_{c}(z)$ is bounded away from zero for large enough $z_{j}$. This together with Proposition 16 will then imply that for a maximally ionized molecule we can find a ball independent of $z$ such that the total number of electrons inside will exceed the total nuclear charge $Z$. For an atom this means that the Radius of Neutrality is bounded above independent of $z$. But for the moment we can only say that the number of electrons outside a ball $B_{r}$ goes to zero uniformly in $\underline{z}$ as $r \rightarrow \infty$.

\section{Lower Bounds to $\varphi, \psi$, and $Q_{c}$}

The aim of this section is to prove that the electrons do not collapse to the point $\mathscr{R}_{j}$ as $z_{j} \rightarrow \infty$. We begin by proving a lower bound to $\varphi$. The major implication of this lower bound is that $\varphi$ is positive in a $z$-independent neighborhood of $\mathscr{R}_{j}$ (see Corollary 18). We use this result to compare the TFW-system to a much simpler boundary value problem to give improved lower bounds to $\varphi$ and $\psi$ near each nucleus. We then extend the lower bound to $\psi$ to a global bound and derive lower bounds to $Q_{c}$ and $\mu$. Finally we use these lower bounds to give the uniform exponential upper bound on $\psi$.

Lemma 17. For all $j=1, \ldots, K$ there exist $\kappa_{j}>0$ and $s_{j}>0$ such that for all $0<\zeta<z_{j}$ and $\kappa>\kappa_{j}$ with $\kappa \zeta^{3 / 2}>s_{j}$,

$$
\varphi(x ; \underline{z} ; N) \geqq \zeta\left|x-\mathscr{R}_{j}\right|^{-1}-\kappa \zeta^{3 / 2}\left|x-\mathscr{R}_{j}\right|^{-1 / 2}
$$

for all $x \in \mathbb{R}^{3}$. 
Proof. Define the functions

$$
h_{\zeta}^{(j)}(x)=\zeta\left|x-\mathscr{R}_{j}\right|^{-1}-\kappa \zeta^{3 / 2}\left|x-\mathscr{R}_{j}\right|^{-1 / 2} .
$$

We will use a comparison argument to prove that $\varphi \geqq h_{\xi}^{(j)}$. Denote $R=(1 / 2)$ $\min _{l \neq k}\left|\mathscr{R}_{l}-\mathscr{R}_{k}\right|$ and let

$$
\Omega_{+}=\left\{x \in \mathbb{R}^{3}|| x-\mathscr{R}_{j} \mid>R \text { for all } j\right\} \text { and } \Omega_{-}=\mathbb{R}^{3} \backslash \Omega_{+} .
$$

Let $S=\left\{x \mid \varphi(x)<h_{\zeta}^{(j)}(x)\right\}$. Since $\zeta<z_{j}$ it is clear that $\mathscr{R}_{i} \notin S$ for all $i=1, \ldots, K$. At infinity we get from Proposition 14 that $\lim _{|x| \rightarrow \infty}|x| \varphi(x)=-Q_{c}(z)>-$ const. Thus $S$ is open and bounded with $\varphi=h_{\zeta}^{(j)}$ on $\partial S$. We will prove that $h_{\zeta}^{(j)}-\varphi$ is subharmonic on $S$, which implies that $S=\emptyset$. We divide the proof of this into two steps.

Step 1. $S \cap \Omega_{+}$.

If $x \in S \cap \Omega_{+}$then from Lemma 13 ,

$$
\begin{aligned}
\Delta \varphi(x)=4 \pi \psi(x)^{2} & \leqq \text { const }\left(\sum_{i=1}^{K} \chi(\alpha)\left|x-\mathscr{R}_{i}\right|^{-4}+\pi^{2} \alpha^{-2}\left|x-\mathscr{R}_{i}\right|^{-2}\right)^{3 / 2} \\
& \leqq \text { const }\left|x-\mathscr{R}_{j}\right|^{-3}
\end{aligned}
$$

where (after minimizing in $\alpha$ ) the constant only depends on the $\mathscr{R}_{i}$. On the other hand

$$
\Delta h_{\zeta}^{(j)}=\frac{1}{4} \kappa \zeta^{3 / 2}\left|x-\mathscr{R}_{j}\right|^{-5 / 2} .
$$

Thus if $\kappa_{\zeta^{3 / 2}}$ is large enough we get $\Delta h_{\zeta}^{(j)}>\Delta \varphi$ on $S \cap \Omega_{+}$.

Step 2. $S \cap \Omega_{-}$.

In this case we estimate $\psi$ by Proposition 8 ,

$$
\Delta \varphi=4 \pi \psi^{2} \leqq 4 \pi\left[\lambda^{-1} \varphi+\lambda^{-1} C(\lambda)\right]_{+}^{3 / 2} .
$$

On $\Omega_{-},\left|x-\mathscr{R}_{j}\right|^{-1 / 2}$ is bounded below. Hence for any fixed $\lambda \in(0,1)$,

$$
\left[\lambda^{-1} h_{\zeta}^{(j)}+\lambda^{-1} C(\lambda)\right]_{+} \leqq \lambda^{-1} \zeta\left|x-\mathscr{R}_{j}\right|^{-1}
$$

if $k \zeta^{3 / 2}$ is large enough (depending on $\lambda$ ). From (51) we obtain for $x \in S \cap \Omega_{-}$,

$$
4 \pi\left[\lambda^{-1} h_{\zeta}^{(j)}+\lambda^{-1} C(\lambda)\right]_{+}^{3 / 2} \leqq 4 \pi \lambda^{-3 / 2} \zeta^{3 / 2}\left|x-\mathscr{R}_{j}\right|^{-3 / 2} \leqq \Delta h_{\zeta}^{(j)}(x),
$$

if $\kappa$ is large enough (depending on $\lambda$ ). Thus on $S \cap \Omega_{-}, \Delta h_{\zeta}^{(j)}(x) \geqq \Delta \varphi(x)$.

Corollary 18. There exist $r_{0}$ with $0<r_{0}<1 / 2 \min _{l \neq k}\left|\mathscr{R}_{l}-\mathscr{R}_{k}\right|$ and $\zeta_{0}>0$ independent of $\underline{z}$ and $N$ such that if $z_{j}>\zeta_{0}$,

$$
\varphi(x ; z ; N)>0 \text { for }\left|x-\mathscr{R}_{j}\right|<r_{0} .
$$

Remark. As $z_{j} \rightarrow 0$ the TFW-solution will converge to the solution without the $j^{\text {th }}$ nucleus. Whether $\varphi$ will be positive or negative near $\mathscr{R}_{j}$ in this case depends on the position of $\mathscr{R}_{j}$ relative to the other nuclei.

For a maximally ionized atom Corollary 18 gives a lower bound to the Radius of Neutrality $R_{Z}$ for large $Z$, since $\varphi\left(R_{Z}\right)<0$. For small $Z$ it is easy to see using the 
results of Benguria and Lieb [2] Sect. 3 that $\lim _{Z \rightarrow 0} R_{Z}=\infty$. Corollary 18 implies that $R_{Z}$ is bounded below by a non-zero constant for all $Z$ (for an upper bound for large $Z$ see Proposition 27).

\subsection{Comparison with Simpler Boundary Value Problem}

With the help of Lemma 17 and Corollary 18 we can now compare the solutions $\varphi$, $\psi$ of the TFW-system to the solutions $y>0, V>0$ of the following much simpler boundary value system:

$$
\begin{array}{lll}
\Delta V=4 \pi\left(V^{3 / 2}-\zeta \delta(x)\right) & \text { on } & \{x|| x \mid<r\}, \\
\Delta y=\left(y^{4 / 3}-\beta V+\mu\right) y & \text { on } & \{x|| x \mid<r\},
\end{array}
$$

and

$$
\left.V\right|_{\{|x|=r\}}=0,\left.\quad y\right|_{\{|x|=r\}}=0,
$$

where $\zeta>0$ and $\beta>0$. Equation (53) is identical to the atomic TF problem with nuclear charge $\zeta$.

Before examining the solution to (53)-(55) we will show how to compare it to the TFW-solution.

Lemma 19. With the notation of Corollary 18 we can for all $C>1$ find $0<r_{C}<r_{0}$ independent of $z$ and $N$ such that if $V_{\zeta}, y_{\zeta}$ are solutions to (53)-(55) with $r=r_{C}$, $\beta=C^{-2}$ and $\zeta_{0}<\zeta<z_{j}$, then on $\left\{x|| x-\mathscr{R}_{j} \mid<r_{C}\right\}$,

$$
\begin{gathered}
\varphi(x ; \underline{z} ; N) \geqq C^{-2} V_{\zeta}\left(x-\mathscr{R}_{j}\right), \\
\psi(x ; z ; N) \geqq y_{\zeta}\left(x-\mathscr{R}_{j}\right) .
\end{gathered}
$$

Proof. Let $\lambda=C^{-1 / 3} \in(0,1)$. It follows from Lemma 17 that we can find $r_{C}$ such that $\left(C^{1 / 3}-1\right) \varphi>C(\lambda)$ on $\left|x-\mathscr{R}_{j}\right|<r_{C}$, where $C(\lambda)$ was defined in Proposition 8 . Hence from Proposition 8 we obtain

$$
\psi(x)^{2} \leqq\left[\lambda^{-1} \varphi+\lambda^{-1} C(\lambda)\right]^{3 / 2} \leqq C \varphi^{3 / 2}
$$

on $\left|x-\mathscr{R}_{j}\right|<r_{C}$. We thus get

$$
\Delta \varphi=4 \pi\left(\psi^{2}-z_{j} \delta\left(x-\mathscr{R}_{j}\right)\right) \leqq 4 \pi\left(C \varphi^{3 / 2}-z_{j} \delta\left(x-\mathscr{R}_{j}\right)\right) .
$$

Let $\tilde{\varphi}_{C}=C^{2} \varphi$ then

$$
\Delta \tilde{\varphi}_{C} \leqq 4 \pi C^{3} \varphi^{3 / 2}-4 \pi C^{2} z_{j} \delta\left(x-\mathscr{R}_{j}\right) \leqq 4 \pi\left(\tilde{\varphi}_{C}^{3 / 2}-z_{j} \delta\left(x-\mathscr{R}_{j}\right)\right) .
$$

A simple comparison argument shows that $V_{\zeta}\left(x-\mathscr{R}_{j}\right) \leqq \tilde{\varphi}_{C}(x)$ on $\left|x-\mathscr{R}_{j}\right|<r_{C}$.

Since $\tilde{\varphi}_{C} \geqq V_{\zeta}>0$ on $\left|x-\mathscr{R}_{j}\right|<r_{C}$ we have

$$
\Delta \psi=\left(\psi^{4 / 3}-\varphi+\mu\right) \psi \leqq\left(\psi^{4 / 3}-C^{-2} V_{\zeta}+\mu\right) \psi .
$$

On the other hand since $\beta=C^{-2}$,

$$
\Delta y_{\zeta}=\left(y_{\zeta}^{4 / 3}-C^{-2} V_{\zeta}+\mu\right) y_{\zeta} \text { for }\left|x-\mathscr{R}_{j}\right|<r_{C}
$$

with $y_{5}=0$ on $\left|x-\mathscr{R}_{j}\right|=r_{C}$. To arrive at $y \leqq \psi$ we appeal to the comparison theorem 
of Hoffmann-Ostenhof [6]. To use this theorem we actually need to know that $y_{\zeta}$ and $V_{\zeta}$ satisfy some regularity properties. These are given in the next theorem.

Theorem 20. For all $r>0, \beta>0$, and $\mu>0$ we have $\zeta_{r, \beta, \mu}>0$ such that for all $\zeta>\zeta_{r, \beta, \mu}$ there exists a unique solution pair $\left(y_{\zeta}, V_{\zeta}\right)$ to $(53)-(55)$ with $y_{\zeta} \in H_{0}^{1}\left(B_{r}\right), y_{\zeta}>0$ for $|x|<r$ and $V_{\zeta} \geqq 0 . y_{\zeta}$ and $V_{\zeta}$ have the following properties:

(i) $y_{\zeta} \in C^{0}(\{|x| \leqq r\})$.

(ii) $y_{\zeta}, V_{\zeta} \in C^{\infty}(\{0<|x|<r\})$.

(iii) $V_{\zeta}>0$ on $\{0<|x|<r\}$ and $\lim _{x \rightarrow 0} V_{\zeta}(x)|x|=\zeta$.

(iv) $V_{\zeta}$ and $y_{\zeta}$ are spherically symmetric and strictly decreasing as functions of $|x|$.

Proof. Equation (53) is the Thomas-Fermi equation. All the stated properties of $V_{\xi}$ can easily be concluded from well known facts of TF-theory (see Lieb [10]). The existence can also be proved by standard ODE-techniques (see Veron [20]).

Equation (54) is studied in exactly the same way as the TFW-equation (Lieb [10]) by considering the functionals

$$
\xi(\varrho)=\int(V \sqrt{\varrho})^{2}+3 / 5 \int \varrho^{5 / 3}-\beta \int V_{\zeta} \varrho+\mu \int \varrho \quad \text { and } \quad \xi^{\prime}(y)=\xi\left(y^{2}\right) .
$$

$\xi^{\prime}$ is defined on $H_{0}^{1}(\{x|| x \mid \leqq r\})$ and $\xi$ is defined on $\left\{\varrho \geqq 0 \mid \sqrt{\varrho} \in H_{0}^{1}\right\}$. That $y_{\zeta}$ is radially decreasing follows from the fact that $V_{\zeta}$ is radially decreasing as in [10, Theorem 7.26]. The only thing we have to prove is that $y_{\zeta} \neq 0$. Choose $\eta \in C_{0}^{\infty}(|x|<r)$ such that $\eta=1$ on $|x|<(1 / 2) r$. We can then prove (Lemma 21 below) that $\lim _{\zeta \rightarrow \infty} \int V_{\xi} \eta=\infty$. It is therefore clear that we can find $\zeta_{\boldsymbol{r}, \beta, \mu}$ such that $\xi(\eta)<0$ for $\zeta>\zeta_{r, \beta, \mu^{*}}$ This implies $y_{\zeta} \neq 0$.

Remark. Since in Theorem 20 we need $\zeta_{r, \beta, \mu}<\zeta$, we see that (57) in Lemma 19 can be used only if $z_{j}>\zeta_{r, \beta, \mu}$ which depends on $C$ and $\mu$. This again shows the necessity of knowing the boundedness of $\mu$ given in the proof of Proposition 29 [case $(Q)$ ] below. Equation (53) by itself of course has a unique solution for all $\zeta>0$.

The next lemma about solutions to the TF-equation is essentially due to Veron [20].

Lemma 21 (Veron). For fixed $r>0$ we have for the solutions $V_{\zeta}$ to the boundary value problems (53)-(55) that

$$
V_{\zeta}(x) \nearrow V_{\infty}^{(r)}(x) \text { as } \zeta>\infty,
$$

for all $x, 0<|x|<r$. The function $V_{\infty}^{(r)}$ satisfies

$$
V_{\infty}^{(r)}(x)=9 \pi^{-2}|x|^{-4}+O\left(|x|^{\sigma}\right),
$$

with $\sigma=-1 / 2+\sqrt{73} / 2$.

Proof. It is clear by comparison that $\zeta_{1}<\zeta_{2}$ implies $V_{\zeta_{1}} \leqq V_{\zeta_{2}}$, and that $V_{\zeta}(x)$ $<9 \pi^{-2}|x|^{-4}$. Thus $\lim _{\zeta \rightarrow \infty} V_{\zeta}=V_{\infty}$ exists pointwise and in the sense of distributions on $0<|x|<r$. Hence we have that $\Delta V_{\infty}=4 \pi V_{\infty}^{3 / 2}$ on $0<|x|<r$. Since $\lim _{x \rightarrow 0}|x| V_{\infty}(x)=\infty$, it follows from Veron [20, Theorem 5.1] that $V_{\infty} \geqq 9 \pi^{-2}|x|^{-4}$ $+O\left(|x|^{\sigma}\right)$. 
Remark. Equations (56) and (59) show that the $\chi(\alpha)$ in the upper bound (42) has the optimal limit $\lim _{\alpha \rightarrow 0} \chi(\alpha)=9 \pi^{-2}$.

Since Lemma 21 gives good control on $V_{\zeta}$, our goal is to estimate $\varphi, \psi$ below in terms of $V_{\zeta}$. For $\varphi$ this was achieved in (56). To get a lower bound to $\psi$ we will use (57), so we have to give a lower bound to $y_{\zeta}$ in terms of $V_{5}$.

Lemma 22. Let $V_{\zeta}, y_{\zeta}$ be the solutions of (53)-(55). If $0<|x|<r$ and $\alpha \in(0,(1-|x| / r)$ $\left.(1+|x| / r)^{-1}\right)$ then,

$$
y_{\zeta}(x)^{4 / 3} \geqq \beta V_{\zeta}((1-\alpha) x)-\pi^{2} \alpha^{-2}(1-\alpha)^{2}|x|^{-2}-\mu .
$$

Proof. Using (34) and (35) we see that for $R<|y|<r-R$,

$$
\beta V_{\zeta}(y) \leqq g_{R} * y_{\zeta}^{4 / 3}(y)+\mu+\pi^{2} R^{-2} .
$$

If we use that $y_{\zeta}$ is radially decreasing we find

$$
\beta V_{\zeta}(y) \leqq y_{\zeta}((|y|-R) y /|y|)^{4 / 3}+\mu+\pi^{2} R^{-2} .
$$

Now choose $R=\alpha|y|$ and $x=(|y|-R) y /|y|=(1-\alpha) y$. Since $0<|x|<R$ and $\alpha \in\left(0,(1-|x| / r)(1+|x| / r)^{-1}\right)$ we clearly get $R<|y|<r-R$.

Corollary 23. For all $C>1, \mu_{0}>0$ we can find $0<r_{C}$ and $0<\zeta_{0}$ such that if $\zeta_{0}<z_{j}$ and $\mu(z ; N) \leqq \mu_{0}$ we get

$$
\psi\left(x+\mathscr{R}_{j} ; \underline{z} ; N\right)^{4 / 3} \geqq C^{-2} V_{\xi}((1-\alpha) x)-\pi^{2} \alpha^{-2}(1-\alpha)^{2}|x|^{-2}-\mu(\underline{z} ; N),
$$

for all $|x|<r_{C} / 2$ and all $\alpha \in(0,1 / 3)$.

Proof. This is just Lemma 19 and Lemma 22.

\subsection{A Global Lower Bound to $\psi$ and Lower Bounds to $Q_{c}$ and $\mu$}

From Corollary 23 and (59) we get good lower bounds for $\psi$ near each nucleus with large nuclear charge. It is not difficult as we will see to extend this to a lower bound for $\psi$ everywhere. Such a lower bound will then in turn imply lower bounds to $Q_{c}$ and to $\mu(z ; N)$. We will first give the proof in the atomic case $(K=1)$, because the molecular case is technically much more complicated.

Proposition 24. (Atomic case: $K=1, \mathscr{R}_{1}=0$ ) For all $r, \mu_{0}>0$ we can find $Z^{(0)}$ such that for all $N \leqq N_{c}, Z$ satisfying $Z>Z^{(0)}$ and $\mu(Z, N) \leqq \mu_{0}$,

$$
\psi(x ; Z ; N) \geqq(3 / 4)^{3 / 4}|x|^{-3 / 2} \exp (-\varepsilon|x|), \text { for }|x| \geqq r,
$$

where $\varepsilon=\max \left\{N-Z, \mu(Z, N)^{1 / 2}\right\}$.

Proof. Since $\psi$ and $\varphi$ are radially symmetric it follows from Newton's theorem that $\varphi(x) \geqq-(N-Z)|x|^{-1}$. Inserting this into the TFW-equation gives

$$
\Delta \psi \leqq \psi^{7 / 3}+\left(\varepsilon|x|^{-1}+\varepsilon^{2}\right) \psi .
$$

We compare $\psi$ with $f(x)=(3 / 4)^{3 / 4}|x|^{-3 / 2} \exp (-\varepsilon|x|)$. For $|x| \neq 0$,

$$
\Delta f(x) \geqq f(x)^{7 / 3}+\left(\varepsilon|x|^{-1}+\varepsilon^{2}\right) f(x) .
$$


Hence $f-\psi$ is subharmonic on $S=\{x \mid f(x)>\psi(x)\}$. It follows from Corollary 23 and Lemma 21 that for any $C>1$ we can find $0<r^{\prime}<\min \left(r, r_{c}\right)$ and $Z^{(0)}$ such that $\left\{|x|=r^{\prime}\right\} \cap S=\emptyset$ for all $Z>Z^{(0)}$ and $\mu(Z, N) \leqq \mu_{0}$. Since $\psi-f \rightarrow 0$ at infinity it follows that $S \cap\left\{|x|>r^{\prime}\right\}=\emptyset$.

We can now prove the lower bounds on $Q$ and $\mu$ in the atomic case. Notice that for the maximal ion $\left(N=N_{c}\right), \mu\left(N_{c}, Z\right)=0$ so $\varepsilon=N-Z=Q_{c}$. For the neutral atom $N=Z$ so $\varepsilon=\mu(Z, Z)$. A general lower bound to $\varepsilon$ thus implies a lower bound to $Q_{c}$ and $\mu_{\text {neutral. }}$. Since $Q_{c}(Z) \rightarrow 0$ as $Z \rightarrow 0$ (see [2]) we of course have to assume that $Z$ is bounded away from 0 .

Theorem 25 (Atomic case). There exists $\delta>0$ such that for all $Z$ large enough and all $N \leqq N_{c}$,

$$
\varepsilon_{N, Z} \equiv \max \left\{N-Z, \mu(Z, N)^{1 / 2}\right\} \geqq \delta .
$$

Proof. It is clearly enough to consider $N, Z$ such that $\mu(N, Z)$ is smaller than some constant. For any $r>0$ we can therefore assume from Proposition 24 that

$$
\begin{aligned}
\int_{|x| \geqq r} \psi(x)^{2} d x & \geqq(3 / 4)^{3 / 2} \int_{|x| \geqq r}|x|^{-3} \exp (-2 \varepsilon|x|) d x \\
& \geqq 4 \pi(3 / 4)^{3 / 2} \int_{2 \varepsilon r}^{1} s^{-1} \exp (-s) d s \geqq-4 \pi(3 / 4)^{3 / 2} e^{-1} \ln (2 \varepsilon r) .
\end{aligned}
$$

Using Proposition 16 we get a bound on $\varepsilon$ for all $r>2$.

Remark. We see that the physically correct exponent $p=5 / 3$ in TFW-theory is critical for the above proof, i.e., the lower bound to $\int_{|x| \geq r} \psi(x)^{2} d x$ is only logarithmically divergent in $\varepsilon$. For $p>5 / 3$ the proof will still work, but for $p<5 / 3$ it would not be a useful method. For the same reason if one tries to give numerical values for the lower bound the result will be quite unsatisfactory.

We now turn to the molecular case. Unfortunately the proof here is technically complicated.

Theorem 26. There exists $\delta>0$ such that if $z_{1}$ (say) is large enough then

$$
\varepsilon \equiv \max \left\{N-Z, \mu(\underline{z} ; N)^{1 / 2}\right\} \geqq \delta .
$$

Proof. The proof is inspired by a proof by L. Jeanneret of Lemma 7.18 in Lieb [10] given in an unpublished note to Haim Brezis. The proof will be divided into several steps.

Step 1. A Lower Bound to $\psi$

As in the atomic case we can assume that $\mu$ is bounded above by some constant. From Corollary 23 we can then assume that $\psi$ is bounded below independent of $\underline{z}$ near $\mathscr{R}_{1}$. That means that we can find $0<r_{1}<(1 / 2) \min _{i \neq j}\left|\mathscr{R}_{i}-\mathscr{R}_{j}\right|$ such that

$$
\psi(x) \geqq \text { const for }\left|x-\mathscr{R}_{1}\right|=r_{1} .
$$

Now from Proposition 8 we know that

$$
\varphi(x) \geqq-\min _{\lambda \in(0,1)} C(\lambda)=-3^{5} 2^{-4} \pi^{2} .
$$


Thus

$$
\Delta \psi \leqq \psi^{7 / 3}+\left(3^{5} 2^{-4} \pi^{2}+\mu\right) \psi .
$$

Just as for the atomic case we can prove that

$$
\psi(x) \geqq C^{\prime}\left|x-\mathscr{R}_{1}\right|^{-3 / 2} \exp \left(-\varepsilon^{\prime}\left|x-\mathscr{R}_{1}\right|\right) \quad \text { on } \quad\left\{x|| x-\mathscr{R}_{1} \mid>r_{1}\right\},
$$

where $\varepsilon^{\prime}=\left(3^{5} 2^{-4} \pi^{2}+\mu\right)^{1 / 2}$. Notice that this bound is not as useful as $(63)$, since it is not given in terms of $\varepsilon$. For simplicity we will from now on assume $\mathscr{R}_{1}=0$.

\section{Step 2. Spherical Average}

Here we use the trick of Lieb $[10$, Lemma 7.17$]$. Let $[\psi]_{a}$ denote the spherical average of $\psi$, i.e.,

$$
[\psi]_{a}(r)=(4 \pi)^{-1} \int_{S^{2}} \psi(r \omega) d \omega .
$$

Define $f(r)=\exp \left([\ln \psi]_{a}(r)\right)$. Then from Jensen's inequality $f(r) \leqq[\psi]_{a}(r)$. Furthermore $f$ has the property (see [10])

$$
[\Delta \psi / \psi]_{a}(r) \geqq \Delta f(r) / f(r) .
$$

Thus for $r>\max _{j}\left|\mathscr{R}_{j}\right|$, again using Newton's theorem we find

$$
\Delta f(r) \leqq\left[\psi^{4 / 3}-\varphi+\mu\right]_{a}(r) f(r) \leqq\left[\psi^{4 / 3}\right]_{a}(r) f(r)+\left(\varepsilon r^{-1}+\varepsilon^{2}\right) f(r) .
$$

From Hölder's inequality we have $\left[\psi^{4 / 3}\right]_{a} \leqq\left[\psi^{2}\right]_{a}^{2 / 3}=[\varrho]_{a}^{2 / 3}$. Defining $g(r)$ $=-\Delta f(r)+[\varrho]_{a}^{2 / 3}(r) f(r)$ we obtain

$$
g(r) \geqq-\left(6 r^{-1}+\varepsilon^{2}\right) f(r) .
$$

Step 3. Choice of Radius

Choose $R>2\left(\max _{j}\left|\mathscr{R}_{j}\right|+1\right)=2\left(\max _{j}\left|\mathscr{R}_{j}-\mathscr{R}_{1}\right|+1\right)>r_{1}$. From (67) we see that

$$
f(R) \geqq C^{\prime} R^{-3 / 2} \exp \left(-\varepsilon^{\prime} R\right) \equiv C_{R} .
$$

Choose $m \in \mathbb{N}$ (depending on $R$ ) such that

$$
(4 / 3) \pi C_{R}^{2}\left(m^{3}-1\right) R^{3}>\int_{|x| \geqq R} \psi(x)^{2} d x .
$$

From Proposition 16 we can choose $m$ independent of $\underline{z}$ and $N$. We claim that there is $R^{\prime} \in(R, m R)$ such that

$$
f^{\prime}\left(R^{\prime}\right)<0 .
$$

If not $f \geqq C_{R}$ on $(R, m R)$ and Hölder's inequality gives

$$
\begin{aligned}
\int_{|x| \geqq R} \psi(x)^{2} d x & \geqq 4 \pi \int_{R}^{m R}[\psi]_{a}^{2}(r) r^{2} d r \\
& \geqq 4 \pi \int_{R}^{m R} f(r)^{2} r^{2} d r \geqq(4 / 3) \pi C_{R}^{2}\left(m^{3}-1\right) R^{3}
\end{aligned}
$$

which is in contradiction with the choice of $m$. 


\section{Step 4. Auxilary Function}

Define for $r \geqq R$ the auxiliary function,

$$
b(r)=\int_{R}^{r} d s s^{-2} \int_{R}^{s}[\varrho]_{a}^{2 / 3}(t) t^{2} d t .
$$

Then

$$
b^{\prime}(r)=r^{-2} \int_{R}^{r}[\varrho]_{a}^{2 / 3}(t) t^{2} d t
$$

and

$$
\Delta b(r)=b^{\prime \prime}(r)+2 r^{-1} b^{\prime}(r)=[\varrho]_{a}^{2 / 3}(r) .
$$

Notice that

$$
\begin{aligned}
b(r) & =\int_{R}^{r}[\varrho]_{a}^{2 / 3}(t) t^{2} \int_{t}^{r} s^{-2} d s d t \leqq\left(\int_{R}^{r}[\varrho]_{a}(t) t^{2} d t\right)^{2 / 3}\left(\int_{R}^{r} t^{-1} d t\right)^{1 / 3} \\
& =\left((4 \pi)^{-1} \int_{|x| \geqq R} \psi(x)^{2} d x\right)^{2 / 3}(\ln (r / R))^{1 / 3} \leqq M_{R}^{2 / 3}(\ln (r / R))^{1 / 3},
\end{aligned}
$$

from Proposition 16 we can choose $M_{R}=(4 \pi)^{-1} M R^{-1 / 2}$. Choose $\eta \in C^{\infty}\left(\overline{\mathbb{R}_{+}}\right)$, $0 \leqq \eta \leqq 1$ with $\eta(t)=1$ for $t \leqq 1$ and $\eta(t)=0$ for $t \geqq 2$. Define $\eta_{n}(x)=\eta(|x| / n)$. For the function $g$ defined in Step 2 we find (with $R^{\prime}$ as in Step 3)

$$
\begin{aligned}
\int_{|x| \geqq R^{\prime}} g(x) \exp (b(x)) \eta_{n}(x) d x= & \int_{|x|=R^{\prime}} e^{b} \eta_{n} \partial_{r} f-f \partial_{r}\left(e^{b} \eta_{n}\right) d S \\
& +\int_{|x| \geqq R^{\prime}} f e^{b}\left\{[\varrho]_{a}^{2 / 3} \eta_{n}-\eta_{n}(\nabla b)^{2}\right. \\
& \left.-\eta_{n} \Delta b-\Delta \eta_{n}-2 \nabla b \nabla \eta_{n}\right\} d x .
\end{aligned}
$$

We assume that $n$ is so large that $\eta_{n}=1$ on $(R, m R)$. If we use that $\partial_{r} f\left(R^{\prime}\right)<0$ (Step 3), $\partial_{r} b\left(R^{\prime}\right) \geqq 0$ and $\Delta b=[\varrho]_{a}^{2 / 3}$ we obtain

$$
\int_{|x| \geqq R^{\prime}} g e^{b} \eta_{n} d x \leqq-\int_{|x| \geqq R^{\prime}} f e^{b}\left\{(\nabla b)^{2} \eta_{n}+\Delta \eta_{n}+2 \nabla b \nabla \eta_{n}\right\} d x .
$$

In the next three steps of the proof we will consider the three terms on the righthand side of (72). We will prove that the first term is bounded by a constant and that the last two terms go to zero as $n \rightarrow \infty$. We begin with the last two terms.

Step 5.

$$
\left|\int_{|x| \geqq R^{\prime}} f e^{b} \Delta \eta_{n} d x\right| \leqq n^{-1 / 2}\left((4 \pi)^{-1} \int_{|x| \geqq R^{\prime}} \psi(x)^{2} d x\right)^{1 / 2}\left(\int_{|x| \geqq 1} e^{2 b(n x)}|\Delta \eta(|x|)|^{2} d x\right)^{1 / 2},
$$

since $\Delta \eta(t)=0$ if $t<1$. Now from Step 4,

$$
2 b(n x) \leqq 2 M_{R}^{2 / 3} \ln (n|x| / R)^{-2 / 3} \ln (n|x| / R) .
$$

Choose $n_{0}$ so large that $2 M_{R}^{2 / 3}\left(\ln \left(n_{0} / R\right)\right)^{-2 / 3}<1 / 2$. Then for $n \geqq n_{0}$,

$$
\begin{aligned}
\left|\int_{|x| \geqq R^{\prime}} f e^{b} \Delta \eta_{n} d x\right| & \leqq n^{-1 / 2} M_{R}^{1 / 2}\left({ }_{|x| \geqq 1}(n|x| / R)^{1 / 2}|\Delta \eta(x)|^{2} d x\right)^{1 / 2} \\
& \leqq \text { const }_{\eta} n^{-1 / 4} M_{R}^{1 / 2} R^{-1 / 4} .
\end{aligned}
$$


Step 6.

$$
\left|\int_{|x| \geqq R^{\prime}} f e^{b} \nabla b \nabla \eta_{n} d x\right| \leqq n^{-1} M_{R}^{1 / 2}\left(\int_{|x| \geqq R^{\prime}}(\nabla b)^{2}(\nabla \eta(|x| / n))^{2} e^{2 b} d x\right)^{1 / 2} .
$$

Now

$$
|\nabla b(r)| \leqq r^{-2}\left(\int_{R}^{r}[\varrho]_{a}(t) t^{2} d t\right)^{2 / 3}\left(\int_{R}^{r} t^{2} d t\right)^{1 / 3} \leqq 3^{-1 / 3} M_{R}^{2 / 3} r^{-1} .
$$

As in Step 5 if $n \geqq n_{0}$,

$$
\int_{|x| \geqq R^{\prime}}(\nabla b)^{2}(\nabla \eta(|x| / n))^{2} e^{2 b} d x \leqq \text { const }_{\eta} M_{R}^{4 / 3} n^{3 / 2} R^{-1 / 2} .
$$

Thus

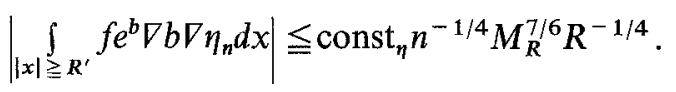

Step 7.

Since $b>0$

$$
\int_{|x| \geqq R^{\prime}} f e^{b}(\nabla b)^{2} \eta_{n} d x \geqq \int_{|x| \geqq m R} f \eta_{n}(\nabla b)^{2} d x .
$$

From (67) we see that $f$ and $[\varrho]_{a}$ are bounded below. Hence from $(70)(\nabla b)^{2}$ is bounded below. If we make sure $n_{0}$ is not too small, there exists $d_{R, m}>0$ such that

$$
\int_{|x| \geqq R^{\prime}} f e^{b}(\nabla b)^{2} \eta_{n_{0}} d x \geqq d_{R, m} .
$$

Going back to (72) we see from (73)-(75) that if $n_{0}$ is large

$$
\int_{|x| \geqq R^{\prime}} g(x) \exp (b(x)) \eta_{n_{0}}(x) d x \leqq-(1 / 2) d_{R, m} .
$$

Step 8. End of Proof

From (68)

$$
\int_{|x| \geqq R^{\prime}} \exp (b(x)) \eta_{n_{0}}(x) f(x)\left(\varepsilon|x|^{-1}+\varepsilon^{2}\right) d x \geqq(1 / 2) d_{R, m} .
$$

But now the final result (66) follows from

$$
\begin{aligned}
& \quad \int_{|x| \geqq R^{\prime}} \exp (b(x)) \eta_{n_{0}}(x) f(x)\left(\varepsilon|x|^{-1}+\varepsilon^{2}\right) d x \\
& \quad \leqq\left(\int_{|x| \geqq R} \psi(x)^{2} d x\right)^{1 / 2}\left(\underset{R \leqq|x| \leqq 2 n_{0}}{\int} \exp (2 b(x))\left(\varepsilon|x|^{-1}+\varepsilon^{2}\right)^{2} d x\right)^{1 / 2} \\
& \leqq C^{(1)}\left(R, n_{0}\right) \varepsilon^{2}+C^{(2)}\left(R, n_{0}\right) \varepsilon,
\end{aligned}
$$

for some constants $C^{(1)}$ and $C^{(2)}$.

\subsection{The Uniform Exponential Decay of $\psi$}

In the atomic case, we will now prove, using the lower bound on $Q_{c}$, that the radius of neutrality $R_{Z}$ for the maximal ion is bounded from above for large $Z$. This result is essentially equivalent to the uniform exponential bound as we will see in the proof of Theorem 28 below. 
Proposition 27. For the maximally ionized atoms there exist $R_{-}, R_{+}>0$ such that

$$
R_{-} \leqq \liminf _{Z \rightarrow \infty} R_{Z} \leqq \limsup _{Z \rightarrow \infty} R_{Z} \leqq R_{+} .
$$

Proof. The lower bound follows from Corollary 18 since $\varphi\left(R_{z}\right)<0$ (see also the remark after the corollary). The upper bound is a consequence of the lower bound on $Q_{c}(Z)$ and the bound on $\int_{|x| \geq r} \psi^{2} d x$ given in Proposition 16. We just have to recall that $\int_{|x| \geqq R_{Z}} \psi(x)^{2} d x=Q_{c}(Z)$.

Remark. For molecules Corollary 18 states that the set $\{x \mid \varphi(x ; \underline{z} ; N)>0\}$ is not too small for large $z$. As for atoms we can now also prove that for the maximally ionized molecule $\{\varphi(x ; z ; N)>0\}$ is bounded for large $z$. This will follow from the proof of the next theorem (see (81) below) in which we give the uniform exponential decay of $\psi$.

Theorem 28. There exist constants $m, \eta>0$ and a radius $R>0$ such that if $z_{1}$ is large enough and $|x| \geqq R$ then

$$
\psi(x ; z ; N) \leqq m \exp \left(-\eta|x|^{1 / 2}\right)
$$

for all $N$.

Proof. From Proposition 16 we can find a radius $R_{1}>\max _{j}\left|\mathscr{R}_{j}\right|+1$ such that $\int_{|x| \geqq R_{1}} \psi^{2} d x \leqq(1 / 2) \delta$, where $\delta$ is the lower bound in Theorem 26. Now consider

$$
\varphi_{R_{1}}(x) \equiv \sum_{j} z_{j}\left|x-\mathscr{R}_{j}\right|-\int_{|y| \leqq R_{1}} \psi(y)^{2}|x-y|^{-1} d y .
$$

Lemma 15 implies

$$
\sup _{|x|=R_{1}} \varphi_{R_{1}}(x) \leqq C R_{1}^{-3 / 2}+\mu .
$$

On $\left\{x|| x \mid>R_{1}\right\}, \varphi_{R_{1}}$ is harmonic and $\varphi_{R_{1}} \rightarrow 0$ as $|x| \rightarrow \infty$. We can thus express $\varphi_{R_{1}}$ using the Poisson integral formula for an exterior domain, i.e., if $|x|>R_{1}$,

$$
\begin{aligned}
\varphi_{R_{1}}(x)= & \left(4 \pi R_{1}\right)^{-1} \int_{|\xi|=R_{1}} \frac{|x|^{2}-R_{1}^{2}}{|x-\xi|^{3}} \varphi_{R_{1}}(\xi) d S_{\xi} \\
\leqq & \left(4 \pi R_{1}\right)^{-1} \int_{|\xi|=R_{1}} \varphi_{R_{1}}(\xi) d S_{\xi}|x|^{-1} \\
& +\left(4 \pi R_{1}\right)^{-1}\left(C R_{1}^{-3 / 2}+\mu\right) \int_{|\xi|=R_{1}}\left|\frac{|x|^{2}-R_{1}^{2}}{|x-\xi|^{3}}-\frac{1}{|x|}\right| d S_{\xi}
\end{aligned}
$$

where $d S_{\xi}$ is the Euclidean measure on $\left\{|\xi|=R_{1}\right\}$. If $|x|>2 R_{1}$ and $|\xi|=R_{1}$ we find

$$
\left|\frac{|x|^{2}-R_{1}^{2}}{|x-\xi|^{3}}-\frac{1}{|x|}\right| \leqq C^{\prime} R_{1}|x|^{-2} .
$$


Furthermore from Newton's theorem

$$
\begin{aligned}
\left(4 \pi R_{1}\right)^{-1} \int_{|\xi|=R_{1}} \varphi_{\boldsymbol{R}_{1}}(\xi) d S_{\xi} & =\sum_{j} z_{j}-\int_{|y| \leqq \boldsymbol{R}_{1}} \psi(y)^{2} d y \\
& =Z-N+\int_{|y| \geqq R_{1}} \psi(y)^{2} d y \leqq Z-N+(1 / 2) \delta .
\end{aligned}
$$

Putting everything together we obtain for $|x| \geqq 2 R_{1}$,

$$
\varphi_{R_{1}}(x) \leqq(Z-N+(1 / 2) \delta)|x|^{-1}+C^{\prime \prime} R_{1}^{1 / 2}|x|^{-2}+C^{\prime} \mu R_{1}^{2}|x|^{-2} .
$$

From (78) we of course have the much simpler estimate

$$
\varphi_{R_{1}}(x) \leqq C R_{1}^{-1 / 2}|x|^{-1}+\mu R_{1}|x|^{-1}, \text { for }|x| \geqq R_{1} .
$$

Indeed (80) is true for $|x|=R_{1}$, for $|x|>R_{1}$ it follows from the maximum principle since both sides are harmonic. The difference between (79) and (80) is that for $N-Z$ large enough (79) will be negative for large $|x|$. We know from (66) that either $N-Z$ $\geqq \delta$ or $\mu^{1 / 2} \geqq \delta$. If $N-Z \geqq \delta$ we use (79) to conclude that for $|x| \geqq 2 R_{1}$,

$$
\varphi(x)-\mu \leqq \varphi_{R_{1}}(x)-\mu \leqq-(1 / 2) \delta|x|^{-1}+C^{\prime \prime} R_{1}^{1 / 2}|x|^{-2}+\mu\left(C^{\prime} R_{1}^{2}|x|^{-2}-1\right) .
$$

If $\mu^{1 / 2} \geqq \delta$ we use (80)

$$
\varphi(x)-\mu \leqq C R_{1}^{-1 / 2}|x|^{-1}+\delta^{2}\left(R_{1}|x|^{-1}-1\right),
$$

for $|x| \geqq 2 R_{1}$. It is now clear that we in both cases can choose $R \geqq 2 R_{1}$ independent of $\underline{z}$ and $N$ (i.e., independent of $\mu$ also) such that

$$
\varphi(x)-\mu \leqq-(1 / 3) \delta|x|^{-1}, \text { for }|x| \geqq R .
$$

From the TFW-equation we obtain for $|x| \geqq R$,

$$
\Delta \psi(x) \geqq \psi(x)^{7 / 3}+(1 / 3) \delta|x|^{-1} \psi(x) \geqq(1 / 3) \delta|x|^{-1} \psi(x) .
$$

We compare $\psi$ with $f=m \exp \left(-2(\delta|x| / 3)^{1 / 2}\right)$. Using Lemma 13 we can choose $m$ such that $\psi(x) \leqq f(x)$ for $|x|=R$. Since $\Delta f(x) \leqq(1 / 3) \delta|x|^{-1} f(x)$ we conclude $\psi \leqq f$ for $|x| \geqq R$.

\section{Compactness}

In the previous sections we have proved pointwise upper and lower bounds to $\psi$ and $\varphi$. We will now study the limit $z_{j} \rightarrow \infty$ for $j=1, \ldots, L$ and $z_{j}$ fixed for $L<j \leqq K$. As in the introduction we will denote this limit by $\underline{z}^{\prime} \rightarrow \infty$. We first have to specify in what sense $N$ tends to infinity. We are interested in two different cases

$$
\begin{gathered}
N<N_{c}(\underline{z}), \quad N-Z \rightarrow Q \quad \text { as } \quad \underline{z}^{\prime} \rightarrow \infty, \\
\mu(z ; N) \rightarrow \mu \text { as } \underline{z}^{\prime} \rightarrow \infty .
\end{gathered}
$$

In this section we will prove that given sequences $\left(N_{n}\right),\left(\underline{z}_{n}\right)$ with $\underline{z}_{n}^{\prime} \rightarrow \infty$ as $n \rightarrow \infty$ and satisfying $(Q)$ or $(\mu)$, then we can find a subsequence $\left(n_{k}\right)$ such that $\psi_{k} \equiv\left(\cdot ; \underline{z}_{n_{k}} ; N_{n_{k}}\right)$ and $\varphi_{k} \equiv \varphi\left(\cdot ; \underline{z}_{n_{k}} ; N_{n_{k}}\right)$ and all their partial derivatives will converge uniformly on all compact sets disjoint from $\left\{\mathscr{R}_{1}, \ldots, \mathscr{R}_{K}\right\}$. Furthermore in case $(Q)$ the 
subsequence can be chosen such that $\mu\left(\underline{z}_{n_{k}} ; N_{n_{k}}\right)$ converges and in case $(\mu)$ such that $N_{n_{k}}-Z_{n_{k}}$ converges. We first prove the last statements.

Proposition 29. In case $(Q)$ we can choose a subsequence such that

$$
\lim _{k \rightarrow \infty} \mu\left(\underline{z}_{n_{k}} ; N_{n_{k}}\right) \text { exists. }
$$

In case $(\mu)$ we can choose a subsequence such that

$$
\lim _{k \rightarrow \infty}\left(N_{n_{k}}-Z_{n_{k}}\right) \text { exists. }
$$

Proof: We have to prove that $\mu_{n}$ is bounded in case $(Q)$ and that $N_{n}-Z_{n}$ is bounded in case $(\mu)$.

Case $(\mu)$

This follows exactly as in [2]. Let

$$
p_{n}(x)=\left(4 \pi \psi_{n}(x)^{2}+\varphi_{n}(x)^{2}\right)^{1 / 2},
$$

then

$$
\Delta p_{n} \geqq 4 \pi\left(\psi_{n}^{10 / 3}+\mu \psi_{n}^{2}\right) p_{n}^{-1} \geqq 0
$$

away from the nuclei. $p_{n}$ is thus subharmonic on the complement of the nuclei. From Proposition 8 and Proposition 12 it follows that $\left|\varphi_{n}\right|$ and $\psi_{n}$ are bounded for $|x|=r$ where $r$ is any radius larger than $\max _{j}\left|\mathscr{R}_{j}\right|$. Hence $p_{n}$ is bounded on $|x|=r$ and since $p_{n}$ is subharmonic

$$
p_{n}(x) \leqq\left(\sup _{|y|=r} p_{n}(y)\right) r /|x| \text { for }|x|>r
$$

thus

$$
\left|N_{n}-Z_{n}\right|=\lim _{|x| \rightarrow \infty}|x| p_{n}(x) \leqq r \sup _{|x|=r} p_{n}(x) \leqq \text { const }
$$

Case $(Q)$

This is more complicated. We can assume that $Q<0$, since if $Q \geqq 0$ we know that $\mu_{n}$ is bounded from Corollary 9 if we recall that $\mu(z ; N)$ is decreasing in $N$.

For fixed $\underline{z}$ the function $N \mapsto E(\underline{z} ; N)$ is convex and decreasing and $\frac{\partial E}{\partial N}=-\mu(z ; N)$. Hence with $Q_{n}=N_{n}-Z_{n}$,

$$
\begin{aligned}
0 & <\mu\left(\underline{z}_{n} ; N_{n}\right)=\mu\left(\underline{z}_{n} ; Z_{n}-\left|Q_{n}\right|\right) \\
& \leqq\left|Q_{n}\right|^{-1}\left(E\left(\underline{z}_{n} ; Z_{n}-2\left|Q_{n}\right|\right)-E\left(\underline{z}_{n} ; Z_{n}\right)\right) .
\end{aligned}
$$

We will prove that $E\left(\underline{z}_{n} ; Z_{n}-2\left|Q_{n}\right|\right)-E\left(\underline{z}_{n} ; Z_{n}\right)$ is bounded as $n \rightarrow \infty$. Without loss of generality we can assume $Q_{n}=Q$. Let $\psi_{\underline{z}_{n}}=\psi\left(\cdot ; \underline{z}_{n} ; Z_{n}\right)$ be the TFW-function corresponding to a neutral molecule. Choose $\eta \in C^{\infty}\left(\overline{\mathbb{R}_{+}}\right), 0 \leqq \eta \leqq 1$, such that $\eta(t)=0$ if $t \leqq 1$ and $\eta(t)=1$ if $t \geqq 2$. For $r<(1 / 2) \min _{i \neq j}\left|\mathscr{R}_{i}-\mathscr{R}_{j}\right|$ we define $\eta_{r}: \mathbb{R}^{3} \rightarrow \mathbb{R}^{3}$ by

$$
\eta_{r}(x)=1-\prod_{j=1}^{K} \eta\left(\left|x-\mathscr{R}_{j}\right| / r\right),
$$


$\eta_{r}$ is 1 near each nuclei. Define $\psi_{z_{n}}^{(r)}=\psi_{z_{n}} \eta_{r}$. We will use $\psi_{z_{n}}^{(r)}$ as a trial function to estimate $E\left(z_{n} ; Z_{n}-2|Q|\right)$. We must show that we can choose $r$ such that $\left\|\psi_{z_{n}}^{(r)}\right\|_{2}^{2}$ $\leqq Z_{n}-2|Q|$. Since $\psi_{z_{n}}$ is the solution for a neutral molecule the corresponding $\mu$ is zero. From Lemma 21 and Corollary 23 we conclude that

$$
\lim _{r \rightarrow 0} \liminf _{n \rightarrow \infty} \int_{\mathbb{R}^{3}}\left(\psi_{\underline{z}_{n}}(x)^{2}-\psi_{\underline{z}_{n}}^{(r)}(x)^{2}\right) d x=\infty .
$$

We can thus choose $r$ such that

$$
\liminf _{n \rightarrow \infty} \int_{\mathbb{R}^{3}}\left(\psi_{\underline{z}_{n}}(x)^{2}-\psi_{\underline{\underline{z}}_{n}}^{(r)}(x)^{2}\right) d x>2|Q|
$$

Then

$$
\limsup _{n \rightarrow \infty} \int_{\mathbb{R}^{3}} \psi_{\underline{z}_{n}}^{(r)}(x)^{2} d x=\limsup _{n \rightarrow \infty} \int_{\mathbb{R}^{3}} \psi_{\underline{z}_{n}}(x)^{2}-\left(\psi_{\underline{z}_{n}}(x)^{2}-\psi_{\underline{z}_{n}}^{(r)}(x)^{2}\right) d x<Z_{n}-2|Q|,
$$

where we have used that $\int \psi_{z_{n}}^{2}=Z_{n}$. From the definition of the energy $E\left(z_{n} ; Z_{n}-2|Q|\right)$ we get that

$$
\mathscr{E}\left(\psi_{z_{n}}^{(r)}\right) \geqq E\left(\underline{z}_{n} ; Z_{n}-2|Q|\right)
$$

for large $n$. Or since $E\left(z_{n} ; Z_{n}\right)=\mathscr{E}\left(\psi_{z_{n}}\right)$

$$
\begin{aligned}
& E\left(\underline{z}_{n} ; Z_{n}-2|Q|\right)-E\left(\underline{z}_{n} ; Z_{n}\right) \leqq \mathscr{E}\left(\psi_{\underline{z}_{n}}^{(r)}\right)-\mathscr{E}\left(\psi_{\underline{z}_{n}}\right) \\
& =\int\left(\eta_{r}^{2}-1\right)\left(\nabla \psi_{z_{n}}\right)^{2}+\psi_{\underline{z}_{n}}^{2}\left(\nabla \eta_{r}\right)^{2}+(1 / 2) \nabla\left(\eta_{r}^{2}\right) \nabla\left(\psi_{\underline{z}_{n}}^{2}\right) d x \\
& \quad+(3 / 5) \int \psi_{\underline{z}_{n}}^{10 / 3}\left(\eta_{r}^{10 / 3}-1\right) d x+\int \varphi_{\underline{z}_{n}} \psi_{\underline{z}_{n}}^{2}\left(1-\eta_{r}^{2}\right)+D\left(\left(1-\eta_{r}^{2}\right) \psi_{\underline{z}_{n}}^{2},\left(1-\eta_{r}^{2}\right) \psi_{z_{n}}^{2}\right) .
\end{aligned}
$$

Since $\eta_{r} \leqq 1$ we arrive at

$$
\begin{aligned}
& E\left(\underline{z}_{n} ; Z_{n}-2|Q|\right)-E\left(\underline{z}_{n} ; Z_{n}\right) \leqq \int\left(\left(\nabla \eta_{r}\right)^{2}-(1 / 2) \Delta\left(\eta_{r}^{2}\right)\right) \psi_{z_{n}}^{2} d x+\int \varphi_{\underline{z}_{n}} \psi_{\underline{z}_{n}}^{2}\left(1-\eta_{r}^{2}\right) d x \\
& +D\left(\left(1-\eta_{r}^{2}\right) \psi_{z_{n}}^{2},\left(1-\eta_{r}^{2}\right) \psi_{z_{n}}^{2}\right) \text {. }
\end{aligned}
$$

Since $1-\eta_{r}^{2}$ is supported away from the nuclei, it follows from Propositions 8, 12 (with $\mu=0$ ) and Theorem 28 that the above quantity is bounded as $n \rightarrow \infty$. Going back to (82) we see that this finishes the proof of the lemma.

We can now prove the main result of this section. In the proof we will need the inequalities of Gagliardo and Nirenberg (Gagliardo [5], Nirenberg [12]).

Lemma 30 (Gagliardo and Nirenberg). If $u, v \in L^{\infty}\left(\mathbb{R}^{n}\right) \cap H^{m}\left(\mathbb{R}^{n}\right)$ and $F \in C^{m}(\mathbb{R})$, with $F(0)=0$, then

$$
\begin{aligned}
\|u v\|_{H^{m}\left(\mathbb{R}^{n}\right)} & \leqq C_{m}\left(\|u\|_{H^{m}\left(\mathbb{R}^{n}\right)}\|v\|_{L^{\infty}\left(\mathbb{R}^{n}\right)}+\|u\|_{L^{\infty}\left(\mathbb{R}^{n}\right)}\|v\|_{H^{m}\left(\mathbb{R}^{n}\right)}\right), \\
\|F(u)\|_{H^{m}\left(\mathbb{R}^{n}\right)} & \leqq C_{m} \sup _{1 \leqq l \leqq m}\left[\left\|F^{(l)}(u)\right\|_{L^{\infty}\left(\mathbb{R}^{n}\right)}\|u\|_{L^{\infty}\left(\mathbb{R}^{n}\right)}^{l-1}\right]\|u\|_{H^{m}\left(\mathbb{R}^{n}\right)} .
\end{aligned}
$$

The main result is

Proposition 31. Given a sequence $\left(N_{n}, \underline{z}_{n}\right)_{n \in \mathbb{N}}$, with $N_{n} \leqq N_{c}(z)$ and $\underline{z}_{n}^{\prime} \rightarrow \infty$ as $n \rightarrow \infty$ and satisfying either $(Q)$ or $(\mu)$, we can then choose a subsequence with the following properties.

(i) The limits $\mu=\lim _{k \rightarrow \infty} \mu_{n_{k}}$ and $Q=\lim _{k \rightarrow \infty}\left(N_{n_{k}}-Z_{n_{k}}\right)$ exist. 
(ii) $0<\psi=\lim _{k \rightarrow \infty} \psi_{n_{k}}$ and $\varphi=\lim _{k \rightarrow \infty} \varphi_{n_{k}}$ exist in the sense of convergence in

$$
C^{\infty}\left(\mathbb{R}^{3} \backslash\left\{\mathscr{R}_{1}, \ldots, \mathscr{R}_{K}\right\}\right),
$$

i.e., $\psi_{n_{k}}, \varphi_{n_{k}}$ and all their partial derivatives converge uniformly on compact subsets. (iii) $\varphi_{n_{k}}$ converges in $L_{\text {loc }}^{3-\varepsilon}\left(\mathbb{R}^{3} \backslash\left\{\mathscr{R}_{1}, \ldots, \mathscr{R}_{L}\right\}\right)$ for all $\varepsilon>0$.

(iv) $\psi_{n_{k}}$ converges in $H^{2}$ on the complement of any compact neighborhood of $\left\{\mathscr{R}_{1}, \ldots, \mathscr{R}_{L}\right\}$.

(v) The limits $\varphi, \psi$, and $\mu$ satisfy

$$
\left.\begin{array}{c}
0=-\Delta \psi+\psi^{7 / 3}-(\varphi-\mu) \psi \\
\Delta \varphi=4 \pi\left(\psi^{2}-\sum_{j=\boldsymbol{L}+1}^{K} z_{j} \delta\left(x-\mathscr{R}_{j}\right)\right)
\end{array}\right\} \text { on } \quad \mathbb{R}^{3} \backslash\left\{\mathscr{R}_{1}, \ldots, \mathscr{R}_{L}\right\}
$$

(vi) For $j=1, \ldots, L, \varphi$ and $\psi$ satisfy

$$
\begin{gathered}
\lim _{x \rightarrow \mathscr{R}_{j}}\left|x-\mathscr{R}_{j}\right|^{4} \varphi(x)=9 \pi^{-2}, \quad \lim _{x \rightarrow \mathscr{R}_{j}}\left|x-\mathscr{R}_{j}\right|^{3} \psi(x)=3 \sqrt{3} \pi^{3 / 2}, \\
\lim _{x \rightarrow \infty}|x| \varphi(x)=-Q .
\end{gathered}
$$

Proof. From Propositions 29 we can assume that (i) is satisfied.

(ii) We will prove that for all open sets $\Omega$ whose closure is compact in $\mathbb{R}^{3} \backslash\left\{\mathscr{R}_{1}, \ldots, \mathscr{R}_{\mathrm{K}}\right\},\left(\psi_{n}\right)$ and $\left(\varphi_{n}\right)$ are bounded sequences in $H^{m}(\Omega)$. We will do this by induction on $m$. From Propositions 8 and 12 we see that $\left(\varphi_{n}\right)$ and $\left(\psi_{n}\right)$ are locally bounded on $\mathbb{R}^{3} \backslash\left\{\mathscr{R}_{1}, \ldots, \mathscr{R}_{K}\right\}$. Hence they are bounded in $H^{0}(\Omega)=L^{2}(\Omega)$. If $\left(\varphi_{n}\right)$, $\left(\psi_{n}\right)$ are bounded in $H^{m}(\widetilde{\Omega})$ for all $\widetilde{\Omega}$ compactly in $\mathbb{R}^{3} \backslash\left\{\mathscr{R}_{1}, \ldots, \mathscr{R}_{\mathrm{K}}\right\}$ we easily conclude from the Gagliardo-Nirenberg inequalities that $\left(\psi_{n}^{7 / 3}\right),\left(\psi_{n}^{2}\right)$, and $\left(\varphi_{n} \psi_{n}\right)$ are bounded in $H^{m}(\widetilde{\Omega})$. Thus $\left(\Lambda \psi_{n}\right)$ and $\left(\Delta \varphi_{n}\right)$ are bounded in $H^{m}(\widetilde{\Omega})$. It follows from standard elliptic estimates that $\left(\varphi_{n}\right)$ and $\left(\psi_{n}\right)$ are bounded in $H^{m+1}(\Omega)$ for a smaller compact set $\Omega$.

This concludes the induction argument. From the Sobolev embedding theorem we get that $\left(\varphi_{n}\right)$ and $\left(\psi_{n}\right)$ are bounded in the topology of $C^{\infty}\left(\mathbb{R}^{3} \backslash\left\{\mathscr{R}_{1}, \ldots, \mathscr{R}_{K}\right\}\right)$. From Ascoli's theorem we conclude (ii). That $0<\psi$ follows as in Step 1 of the proof of Theorem 26.

(iii) We only have to prove that $\left(\varphi_{n_{k}}\right)$ converges in $L^{3-\varepsilon}$ near each nucleus $\mathscr{R}_{j}$, $j=L+1, \ldots, K$. Since we know that $\left(\varphi_{n_{k}}\right)$ converges pointwise away from the nuclei, we can conclude the $L^{3-\varepsilon}$-convergence from the dominated convergence theorem,

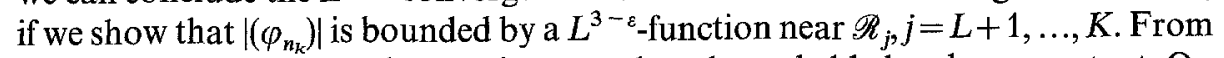
Proposition 8 we know that $\varphi_{n_{k}}$ is everywhere bounded below by a constant. On the other hand for $r<\min _{i \neq j}\left|\mathscr{R}_{i}-\mathscr{R}_{j}\right|, \varphi_{n_{k}}$ is bounded above by a constant $C_{j}$ on $\left|x-\mathscr{R}_{j}\right|=r$ (see Proposition 12). Thus $\varphi_{n_{k}} \leqq\left(z_{j}+1\right)\left|x-\mathscr{R}_{j}\right|^{-1}+C_{j}$ both near $\mathscr{R}_{j}$ and for $\left|x-\mathscr{R}_{j}\right|=r$. Now

$$
\varphi_{n_{k}}-\left(\left(z_{j}+1\right)\left|x-\mathscr{R}_{j}\right|^{-1}+C_{j}\right)
$$

is subharmonic on $\left\{0<\left|x-\mathscr{R}_{j}\right|<r\right\}$. Hence

$$
\varphi_{n_{k}} \leqq\left(z_{j}+1\right)\left|x-\mathscr{R}_{j}\right|^{-1}+C_{j} \text { for }\left|x-\mathscr{R}_{j}\right|<r
$$

and the $L^{3-\varepsilon}$-convergence follows. 
(iv) For $j=L+1, \ldots, K$ we have on $\left\{\left|x-\mathscr{R}_{j}\right| \leqq r\right\}[r$ chosen in (iii) $]$ from the TFW-equation,

$$
\Delta \psi_{n_{k}} \geqq\left(-\left(z_{j}+1\right)\left|x-\mathscr{R}_{j}\right|^{-1}-C_{j}\right) \psi_{n_{k}} .
$$

We compare $\psi_{n_{k}}$ with $f(x)=c \exp \left(-(1 / 2)\left(z_{j}+2\right)\left|x-\mathscr{R}_{j}\right|\right)$ which satisfies

$$
\Delta f=\left((1 / 4)\left(z_{j}+2\right)^{2}-\left(z_{j}+2\right)\left|x-\mathscr{R}_{j}\right|^{-1}\right) f .
$$

If we choose $r_{j}<\left((1 / 2)\left(z_{j}+2\right)^{2}+C_{j}\right)^{-1}$, then for $\left|x-\mathscr{R}_{j}\right|<r_{j}$,

$$
\Delta f \leqq\left(-C_{j}-\left(z_{j}+1\right)\left|x-\mathscr{R}_{j}\right|^{-1}\right) f .
$$

As $k \rightarrow \infty$ we know that since $z_{j}$ is fixed $\psi_{n_{k}}$ is bounded by a constant on $\left\{\left|x-\mathscr{R}_{j}\right|=r_{j}\right\}$ (see Lemma 13). We can thus choose $c$ such that $w_{n_{k}} \leqq f$ for $\left|x-\mathscr{R}_{j}\right|=r_{j}$. From (88) and (89) it then follows by a comparison argument that

$$
\psi_{n_{k}}(x) \leqq c \exp \left(-(1 / 2)\left(z_{j}+2\right)\left|x-\mathscr{R}_{j}\right|\right) \text { for }\left|x-\mathscr{R}_{j}\right| \leqq r_{j} .
$$

Especially $\psi_{n_{k}}$ is bounded by a constant near each $\mathscr{R}_{j}, j=L+1, \ldots, K$. This together with the exponential bound in Theorem 28 and the dominated convergence theorem imply the $L^{2}$-convergence of $\psi_{n_{k}}$. (iv) then follows from the TFWequation.

(v) It is now clear that $\left(\varphi_{n_{k}}\right),\left(\psi_{n_{k}}\right),\left(\psi_{n_{k}}^{7 / 3}\right),\left(\psi_{n_{k}}^{2}\right)$, and $\left(\varphi_{n_{k}} \psi_{n_{k}}\right)$ converge in the sense of distributions on $\mathscr{R}^{3} \backslash\left\{\mathscr{R}_{1}, \ldots, \mathscr{R}_{L}\right\}$. Since (85) holds for $\varphi_{n_{k}}, w_{n_{k}}$, and $\mu_{n_{k}}$ it holds in the limit as well.

(vi) The bounds

$$
\lim _{x-\mathscr{R}_{j}}\left|x-\mathscr{R}_{j}\right|^{4} \varphi(x) \leqq 9 \pi^{-2} \text { and } \limsup _{x \rightarrow \mathscr{R}_{j}}\left|x-\mathscr{R}_{j}\right|^{3} \psi(x) \leqq 3 \sqrt{3} \pi^{3 / 2}
$$

follow from Propositions 8 and 12. The lower bounds

$$
\liminf _{x \rightarrow \mathscr{R}_{j}}\left|x-\mathscr{R}_{j}\right|^{4} \varphi(x) \geqq 9 \pi^{-2} \text { and } \liminf _{x \rightarrow \mathscr{R}_{j}}\left|x-\mathscr{R}_{j}\right|^{3} \psi(x) \geqq 3 \sqrt{3} \pi^{3 / 2}
$$

follow from Lemmas 19, 21 and Corollary 23.

To prove (87) we write as in Theorem 28 for $|x| \geqq R_{1}$,

$$
\varphi_{n}(x)=\left(\varphi_{R_{1}}\right)_{n}(x)+\int_{|y| \geqq R_{1}} \psi_{n}(y)^{2}|x-y|^{-1} d y .
$$

Using the Poisson integral formula as in Theorem 28 , we get for $|x| \geqq 2 R_{1}$,

$$
\begin{gathered}
\left.\left|\varphi_{n}(x)-\left(Z_{n}-N_{n}\right)\right| x\right|^{-1} \mid \\
\leqq \int_{|y| \geqq R_{1}} \psi_{n}(y)^{2}\left(|x|^{-1}+|x-y|^{-1}\right) d y+(4 \pi)^{-1} C^{\prime}|x|^{-2} \int_{|\xi|=R_{1}}\left|\left(\varphi_{R_{1}}\right)_{n}(\xi)\right| d S_{\xi} .
\end{gathered}
$$

From the uniform exponential decay (77) and the bound on $\varphi$ we see that given $\varepsilon>0$ we can choose $R_{1}$ such that for some constant $C$ and $|x|>2 R_{1}$,

$$
\left.\left.|x|\left|\varphi_{n}(x)-\left(Z_{n}-N_{n}\right)\right| x\right|^{-1}|\leqq \varepsilon+C| x\right|^{-1} .
$$

If we let $n \rightarrow \infty$ we find

$$
\| x|\varphi(x)+Q| \leqq \varepsilon+(4 \pi)^{-1} C^{\prime} C_{R_{1}}|x|^{-1} .
$$




\section{Asymptotic Expansions Near the Nuclei}

Our goal is to prove that given $\mu \geqq 0$ then $\varphi, \psi$, and $Q$ are uniquely determined from (85)-(87). Likewise given $Q$ then $\varphi, \psi$, and $\mu$ are uniquely determined. This together with Proposition 31 will then imply Theorems 1 and 4 . In this section we will present the first step which is to prove that $\varphi$ and $\psi$ satisfy the asymptotic expansions in Theorem 6. For technical reasons it will be necessary for us to keep track of the asymptotic expansion of the function

$$
W(x)=\psi(x)^{4 / 3}-\varphi(x) .
$$

Define the functions

$$
\begin{aligned}
& \varphi_{n}(x)=|x|^{-4}\left(a_{1}+a_{2}|x|^{2}+\ldots+a_{n}|x|^{2(n-1)}\right), \quad n \geqq 1, \\
& \psi_{n}(x)=|x|^{-3}\left(b_{1}+b_{2}|x|+\ldots+b_{n}|x|^{2(n-1)}\right), \quad n \geqq 1, \\
& W_{n}(x)=|x|^{-2}\left(c_{1}+c_{2}|x|^{-2}+\ldots+c_{n-1}|x|^{2(n-2)}\right), \quad n \geqq 2, \quad W_{1} \equiv 0,
\end{aligned}
$$

where $a_{n}, b_{n}$, and $c_{n}$ are uniquely defined by requiring $a_{1}, b_{1} \neq 0$ and that asymptotically near $x=0$,

$$
\begin{gathered}
\Delta \psi_{n}(x)=\psi_{n}(x) W_{n}(x)+O\left(|x|^{2 n-7}\right), \\
\Delta \varphi_{n}(x)=4 \pi \psi_{n}(x)^{2}+O\left(|x|^{2 n-6}\right), \\
W_{n}(x)=\psi_{n}(x)^{4 / 3}-\varphi_{n}(x)+O\left(|x|^{2 n-4}\right) .
\end{gathered}
$$

It is not hard to see that $a_{1}=9 \pi^{-2}, b_{1}=3 \sqrt{3} \pi^{-3 / 2}$, and that for $n \geqq 1$,

$$
\begin{gathered}
\Delta \psi_{n}-\psi_{n} W_{n}=c_{n} b_{1}|x|^{2 n-7}+O\left(|x|^{2 n-5}\right), \\
\Delta \varphi_{n}-4 \pi \psi_{n}^{2}=\left(8 \pi b_{n+1} b_{1}-(2 n-3)(2 n-4) a_{n+1}\right)|x|^{2 n-6}+O\left(|x|^{2 n-4}\right), \\
W_{n}-\left(\psi_{n}^{4 / 3}-\varphi_{n}\right)=\left((4 / 3) b_{1} b_{n+1}-c_{n}-a_{n+1}\right)|x|^{2 n-4}+O\left(|x|^{2 n-2}\right) .
\end{gathered}
$$

From this we can compute all the $a$ 's, $b$ 's, and $c$ 's. $\varphi_{4}$ and $\psi_{4}$ are the functions given in Theorem 6.

Proposition 32. If $\varphi, \psi \in C^{\infty}(\{x \mid 0<x<R\})$ satisfy

$$
\begin{gathered}
-\Delta \psi+\psi^{7 / 3}-\varphi \psi+\mu \psi=0, \\
\Delta \varphi=4 \pi \psi^{2},
\end{gathered}
$$

and

$$
\lim _{x \rightarrow 0} \varphi(x)|x|^{4}=a_{1} \quad \text { and } \quad \lim _{x \rightarrow 0} \psi(x)|x|^{3}=b_{1},
$$

then

$$
\varphi(x)-\mu=\varphi_{4}(x)+O\left(|x|^{\sigma}\right), \quad \psi(x)=\psi_{4}(x)+O\left(|x|^{\sigma+1}\right),
$$

and

$$
W(x)+\mu=W_{5}(x)+O\left(|x|^{\sigma+2}\right),
$$

where $\sigma=1 / 2+\sqrt{73} / 2$. 
Remark. We can compute

$$
W_{5}(x)=6|x|^{2}+(3 / 8) \pi^{2}+\left(\pi^{4} / 32\right)|x|^{2}-\left(7 \pi^{6} / 1152\right)|x|^{4} .
$$

Strategy of Proof of Proposition 32. Without loss of generality we can assume that $\mu=0$. We formulate the proof as an induction argument. We assume that for some $n, 1 \leqq n \leqq 5$, the following estimates hold:

$$
\begin{gathered}
\varphi(x)-\varphi_{n}(x)=o\left(|x|^{2 n-6}\right)+O\left(|x|^{\sigma}\right), \\
\psi(x)-\psi_{n}(x)=o\left(|x|^{2 n-5}\right)+O\left(|x|^{\sigma+1}\right), \\
W(x)-W_{n}(x)=o\left(|x|^{2 n-6}\right)+O\left(|x|^{\sigma+2}\right) .
\end{gathered}
$$

We then prove that they hold for $n$ replaced by $n+1$. First notice that (101)-(103) hold for $n=1$. In fact (101) and (102) are equivalent to (100), (103) follows from (101) and (102).

In proving the induction step it is important to proceed in the right order. First we prove (103) for $n$ replaced by $n+1$. Using (103) for $n+1$ we then prove (101) for $n+1$. (102) is then a trivial consequence of (103) and (101). Before proving (103) we have to settle an important technical point.

Lemma 33. If (101)-(103) hold for some $n, 1 \leqq n \leqq 6$, then

$$
\nabla \varphi(x)=\nabla \varphi_{n}(x)+o\left(|x|^{2 n-7}\right)+O\left(|x|^{\sigma-1}\right),
$$

and

$$
\nabla \psi(x)=\nabla \psi_{n}(x)+o\left(|x|^{2 n-8}\right)+O\left(|x|^{\sigma}\right) .
$$

Remark. While (104) has the right power law behavior compared to (101), (105) is very bad compared to (102), unless $n=6$. Since we eventually will prove (101) $-(103)$ for $n=6$ this defect is unimportant, see also Corollary 36 below.

Proof. Choose $\eta \in C_{0}^{\infty}\left(\mathbb{R}_{+}\right), 0 \leqq \eta \leqq 1$ with supp $\eta \subseteq(1 / 2,5 / 2)$ and $\eta=1$ on $(1,2)$. For $0<r<(2 / 5) R$, let $\eta_{r} \in C_{0}^{\infty}(\{0<|x|<R\})$ be defined by $\eta_{r}(x)=\eta(|x| / r)$. For all $f \in C_{0}^{\infty}(\{0<|x|<R\})$ define $f_{r}=f \eta_{r}$. Then by a trivial identification $f_{r} \in C_{0}^{\infty}\left(\mathbb{R}^{3}\right)$ and

$$
\Delta f_{r}=f \Delta \eta_{r}+2 \nabla f \nabla \eta_{r}+\eta_{r} \Delta f \in C_{0}^{\infty}\left(\mathbb{R}^{3}\right) .
$$

Thus by integrating by parts

$$
f_{r}(x)=-(4 \pi)^{-1}\left(\Delta f_{r}\right) *|x|^{-1}=-(4 \pi)^{-1}\left[\left(-f \Delta \eta_{r}+\eta_{r} \Delta f\right) *|x|^{-1}-2\left(f \nabla \eta_{r}\right) * x /|x|^{3}\right] .
$$

Differentiating with respect to $x$ we obtain for $x \notin \operatorname{supp}\left(\nabla \eta_{r}\right)$,

$$
\begin{aligned}
4 \pi \nabla f_{r}(x)= & \left(\eta_{r} \Delta f-f \Delta \eta_{r}\right) * x /|x|^{3}+2\left(f \nabla \eta_{r}\right) * 1 /|x|^{3} \\
& -6 \int f(y)(x-y) \cdot \nabla \eta_{r}(y)|x-y|^{-5}(x-y) d y .
\end{aligned}
$$

Since $x \notin \operatorname{supp}\left(\nabla \eta_{r}\right)$ we do not pick up delta functions from differentiating $x /|x|^{3}$. For $f$ we will insert $\varphi-\varphi_{n}$ and $\psi-\psi_{n}$. Notice that

$$
\Delta\left(\varphi-\varphi_{n}\right)=4 \pi\left(\psi^{2}-\psi_{n}^{2}\right)+O\left(|x|^{2 n-6}\right)=o\left(|x|^{2 n-8}\right)+O\left(|x|^{\sigma-2}\right)
$$


from (93) and (101) (which we assume holds for $n$ ). Likewise

$$
\Delta\left(\psi-\psi_{n}\right)=W \psi-W_{n} \psi_{n}+O\left(|x|^{2 n-7}\right)=o\left(|x|^{2 n-9}\right)+O\left(|x|^{\sigma-1}\right) .
$$

Using $\varphi-\varphi_{n}=o\left(|x|^{2 n-6}\right)+O\left(|x|^{\sigma}\right)$, with $(5 / 4) r \leqq|x| \leqq(7 / 4) r$ gives

$$
\begin{aligned}
&\left|\nabla\left(\varphi-\varphi_{n}\right)(x)\right|=\left|\nabla\left(\varphi-\varphi_{n}\right)_{r}(x)\right| \\
& \leqq {\left[o\left(r^{2 n-6}\right)+O\left(r^{\sigma}\right)\right]\left(\int \left|\Delta \eta_{r}(y)\left\|x-\left.y\right|^{-2}+\left|\nabla \eta_{r}(y) \| x-y\right|^{-3}\right)\right.\right.} \\
&+\left[o\left(r^{2 n-8}\right)+O\left(r^{\sigma-2}\right)\right] \int \eta_{r}(y)|x-y|^{-2} d y \\
& \leqq o\left(r^{2 n-7}\right)+O\left(r^{\sigma-1}\right)=o\left(|x|^{2 n-7}\right)+O\left(|x|^{\sigma-1}\right)
\end{aligned}
$$

Likewise for $\psi-\psi_{n}=o\left(|x|^{2 n-5}\right)+O\left(|x|^{\sigma+1}\right)$,

$$
\left|\nabla\left(\psi-\psi_{n}\right)(x)\right| \leqq o\left(r^{2 n-8}\right)+O\left(r^{\sigma}\right)=o\left(|x|^{2 n-8}\right)+O\left(|x|^{\sigma}\right)
$$

Lemma 34. If (101)-(103) hold for $n, 1 \leqq n \leqq 5$, then (103) holds for $n$ replaced by $n+1$.

Proof. We have to prove that

$$
W(x)-W_{n}(x)=c_{n}|x|^{2 n-4}+o\left(|x|^{2 n-4}\right)+O\left(|x|^{\sigma+2}\right) .
$$

For any $\varepsilon>0$ we will prove that there exists $0<R_{\varepsilon}<R$ such that for $|x|<R_{\varepsilon}$,

$$
\left(c_{n}-2 \varepsilon\right)|x|^{2 n-4}<W(x)-W_{n}(x)+O\left(|x|^{\sigma+2}\right)<\left(c_{n}+2 \varepsilon\right)|x|^{2 n-4} .
$$

We concentrate on the lower bound, the upper bound is proved in exactly the same way. For $0<r<R$ define

$$
a_{r}^{\varepsilon}=\left(c_{n}-\varepsilon\right)^{-1} \sup _{|x| \leqq r}\left(|x|^{6-2 n}\left|W(x)-W_{n}(x)\right|+\left|c_{n}-\varepsilon\right| r^{2}\right)
$$

From $(103)_{n}$ we get that $\lim _{r \rightarrow 0} a_{r}^{\varepsilon}=0$. Define for $k \geqq 0$,

$$
f_{r}^{\varepsilon}=\left(W(x)-W_{n}(x)\right)|x|^{6-2 n}+\left(c_{n}-\varepsilon\right)\left(-|x|^{2}+a_{r}^{\varepsilon}(|x| / r)^{3}\right)+k|x|^{\sigma+8-2 n}
$$

It is only relevant to have $k \neq 0$ if $n=5$. Our aim is to prove that we can find $0<r_{\varepsilon}<R$ such that $f_{r_{\varepsilon}}^{\varepsilon}(x) \geqq 0$ for $|x| \leqq r_{\varepsilon}$. The final result (106) will then easily follow. From the definition of $a_{r}^{\varepsilon}$ we get $f_{r}^{\varepsilon}(x) \geqq 0$ for $|x|=r$ and from $(103)_{n} f_{r}^{\varepsilon}(x) \rightarrow 0$ as $x \rightarrow 0$. Thus either $f_{r}^{\varepsilon}(x) \geqq 0$ for all $|x| \leqq r$ or there exists $x_{0}, 0<\left|x_{0}\right|<r$ such that

$$
f_{r}^{\varepsilon}\left(x_{0}\right)<0, \quad \nabla f_{r}^{\varepsilon}\left(x_{0}\right)=0 \text { and } \Delta f_{r}^{\varepsilon}\left(x_{0}\right) \geqq 0 \text {. }
$$

We prove that for $r$ small enough (109) cannot occur.

$$
\begin{aligned}
\nabla f_{r}^{\varepsilon}(x)= & \left(\nabla W-\nabla W_{n}\right)|x|^{6-2 n}+(6-2 n)\left(W-W_{n}\right)|x|^{4-2 n} x \\
& +\left(c_{n}-\varepsilon\right)\left(-2 x+3 a_{r}^{\varepsilon}(|x| / r)^{3}|x|^{-2} x\right)+O\left(|x|^{\sigma+7-2 n}\right) \\
= & \left(\frac{4}{3} \psi(x)^{1 / 3} \nabla \psi(x)-\nabla \varphi(x)-\nabla W_{n}(x)\right)|x|^{6-2 n}+(6-2 n)\left(W-W_{n}\right)|x|^{4-2 n} x \\
& +\left(c_{n}-\varepsilon\right)\left(-2 x+3 a_{r}^{\varepsilon}(|x| / r)^{3}|x|^{-2} x\right)+O\left(|x|^{\sigma+7-2 n}\right)
\end{aligned}
$$

Here $o(\cdot)$ and $O(\cdot)$ are vector-valued functions that are independent of $r$. Even though (105) gives an unsatisfactory estimate for $\nabla \psi$, we can get a good estimate for 
$\nabla \psi\left(x_{0}\right)$ by using the above expression together with (103), (104), and (109). We get

$$
\begin{aligned}
\frac{4}{3} \psi\left(x_{0}\right)^{1 / 3} \nabla \psi\left(x_{0}\right)= & \nabla \varphi_{n}\left(x_{0}\right)+\nabla W_{n}\left(x_{0}\right)+O\left(\left|x_{0}\right|^{\sigma-1}\right)+o\left(\left|x_{0}\right|^{2 n-7}\right) \\
& +a_{r}^{\varepsilon}\left(\left|x_{0}\right| / r\right)^{3} O\left(\left|x_{0}\right|^{2 n-7}\right) \\
= & \frac{4}{3} \psi_{n}\left(x_{0}\right)^{1 / 3} \nabla \psi_{n}\left(x_{0}\right)+o\left(\left|x_{0}\right|^{2 n-7}\right) \\
& +a_{r}^{\varepsilon}\left(\left|x_{0}\right| / r\right)^{3} O\left(\left|x_{0}\right|^{2 n-7}\right)+O\left(\left|x_{0}\right|^{\sigma-1}\right),
\end{aligned}
$$

here we have used (94).

We know that $W-W_{n}=o\left(|x|^{2 n-6}\right)$, thus since $\nabla f_{r}^{\varepsilon}\left(x_{0}\right)=0$ we get from the first equality in (110),

$$
\partial_{r} W\left(x_{0}\right)-\partial_{r} W_{n}\left(x_{0}\right)=o\left(\left|x_{0}\right|^{2 n-7}\right)+a_{r}^{\varepsilon}\left(\left|x_{0}\right| / r\right)^{3} O\left(\left|x_{0}\right|^{2 n-7}\right)+O\left(\left|x_{0}\right|^{\sigma+1}\right) .
$$

Inserting this and (111) into the last inequality of (109) gives

$$
\begin{aligned}
0 \leqq & \frac{4}{9} \psi_{n}\left(x_{0}\right)^{-2 / 3}\left(\nabla \psi_{n}\left(x_{0}\right)\right)^{2}+\frac{4}{3} \psi_{n}^{4 / 3} W_{n}\left(x_{0}\right)-4 \pi \psi_{n}\left(x_{0}\right)^{2}-\Delta W_{n}\left(x_{0}\right) \\
& +\frac{4}{3} \psi\left(W\left(x_{0}\right)-W_{n}\left(x_{0}\right)\right)+\left(a_{r}^{\varepsilon}\right)^{2}\left(\left|x_{0}\right| / r\right)^{6} O\left(\left|x_{0}\right|^{4 n-10}\right) \\
& +a_{r}^{\varepsilon}\left(\left|x_{0}\right| / r\right)^{3} O\left(\left|x_{0}\right|^{2 n-8}\right)+o\left(\left|x_{0}\right|^{2 n-8}\right)+O\left(\left|x_{0}\right|^{\sigma-2}\right),
\end{aligned}
$$

where we have used (102) . Now (95)-(97) easily imply that

$$
\Delta W_{n}=\frac{4}{9} \psi_{n}^{-2 / 3}\left(\nabla \psi_{n}\right)^{2}+\frac{4}{3} \psi_{n}^{4 / 3} W_{n}-4 \pi \psi_{n}^{2}+\frac{4}{3} c_{n} b_{1}^{4 / 3}|x|^{2 n-8}+O\left(|x|^{2 n-6}\right) .
$$

Since $\left|x_{0}\right| \leqq r$ and $a_{r}^{\varepsilon} \rightarrow 0$ as $r \rightarrow 0$ we can finally write

$$
0 \leqq-\frac{4}{3} c_{n} b_{1}^{4 / 3}+\frac{4}{3} \psi\left(x_{0}\right)^{4 / 3}\left(W\left(x_{0}\right)-W_{n}\left(x_{0}\right)\right)\left|x_{0}\right|^{8-2 n}+o_{r}(1)+O\left(\left|x_{0}\right|^{\sigma+6-2 n}\right) .
$$

Here $o_{r}(1)$ is a function of $r$ that goes to zero as $r \rightarrow 0$. We now use the definition (108) of $f_{r}^{\varepsilon}$ and (102) for $n=1$,

$$
\begin{aligned}
\frac{4}{3} c_{n} b_{1}^{4 / 3} \leqq & {\left[\frac{4}{3} b_{1}^{4 / 3}+o_{x_{0}}(1)\right]\left(\left(c_{n}-\varepsilon\right)\left(1-a_{r}^{\varepsilon}\left(\left|x_{0}\right| / r\right)^{3}\left|x_{0}\right|^{-2}\right)-k\left|x_{0}\right|^{\sigma+6-2 n}\right) } \\
& +o_{\mathbf{r}}(1)+O\left(\left|x_{0}\right|^{\sigma+6-2 n}\right) .
\end{aligned}
$$

We have used that $f_{r}^{\varepsilon}\left(x_{0}\right)<0$ and assumed $r$ so small that $\frac{4}{3} b_{1}^{4 / 3}+o_{x_{0}}(1)>0$. From the definition of $a_{r}^{\varepsilon}$ it is clear that $\left(c_{n}-\varepsilon\right) a_{r}^{\varepsilon} \geqq 0$, hence

$$
\frac{4}{3} b_{1}^{4 / 3} \varepsilon \leqq-\left[\frac{4}{3} b_{1}^{4 / 3}+o_{x_{0}}(1)\right] k\left|x_{0}\right|^{\sigma+6-2 n}+o_{r}(1)+O\left(\left|x_{0}\right|^{\sigma+6-2 n}\right) .
$$

It is therefore clear that we can choose $r_{\varepsilon}$ and in the case $n=5, k$, such that (109) never can be satisfied for $\left|x_{0}\right|<r_{\varepsilon}$, i.e., $f_{r_{\varepsilon}}^{\varepsilon}(x) \geqq 0$ on $|x| \leqq r_{\varepsilon}$ or

$$
W(x)-W_{n}(x) \geqq\left(c_{n}-\varepsilon\right)|x|^{2 n-4}\left(1-a_{r_{\varepsilon}}^{\varepsilon} r_{\varepsilon}^{-3}|x|\right)+O\left(|x|^{\sigma+2}\right) .
$$

If we choose $R_{\varepsilon}=\min \left\{r_{\varepsilon}, \varepsilon r_{\varepsilon}^{3}\left(a_{r_{\varepsilon}}^{\varepsilon}\left(c_{n}-\varepsilon\right)\right)^{-1}\right\}$ (recall that $\left(c_{n}-\varepsilon\right) a_{r}^{\varepsilon} \geqq 0$ ), then for all $|x| \leqq R_{\varepsilon}$

$$
\left(c_{n}-\varepsilon\right) a_{r_{\varepsilon}}^{\varepsilon} r_{\varepsilon}^{-3}|x| \leqq \varepsilon .
$$

Thus for $0<|x| \leqq R_{\varepsilon}$,

$$
W(x)-W_{n}(x) \geqq\left(c_{n}-2 \varepsilon\right)|x|^{2 n-4}+O\left(|x|^{\sigma+2}\right) .
$$

Lemma 35. If (101)-(102) hold for $n, 1 \leqq n \leqq 5$, then (101) holds for $n$ replaced by $n+1$. 
Proof. Since $\sigma<4$, (101) for $n=5$ is equivalent to (101) for $n=6$. We thus only have to consider $n \leqq 4$. As a consequence of Lemma 34 we are allowed to use (103) for $n$ replaced by $n+1$, i.e., $(103)_{n+1}$.

Let $\tilde{\varphi}_{n}=\varphi_{n}-\varphi_{1}$. We first prove

$$
\psi^{2}=\left(\varphi-\tilde{\varphi}_{n}\right)^{3 / 2}+(4 \pi)^{-1} \Delta \tilde{\varphi}_{n}+l_{n}|x|^{2 n-6}+o\left(|x|^{2 n-6}\right),
$$

where

$$
\begin{aligned}
l_{n} & =\frac{3}{2} a_{1}^{1 / 2} c_{n}+(4 \pi)^{-1}\left[(2 n-3)(2 n-4) a_{n+1}-6 \pi\left(a_{n+1}+c_{n}\right) a_{1}^{1 / 2}\right] \\
& =(4 \pi)^{-1}(2 n-3)(2 n-4) a_{n+1}-\frac{3}{2} a_{1}^{1 / 2} a_{n+1} .
\end{aligned}
$$

Notice that $l_{1}=(3 / 2) a_{1}^{1 / 2} c_{1}$. For $n=1, \tilde{\varphi}_{1}=0$ and

$$
\begin{aligned}
\psi^{2}=(\varphi+W)^{3 / 2} & =\varphi^{3 / 2}+\frac{3}{2} \varphi^{1 / 2} W+\varphi^{3 / 2} O\left(W^{2} / \varphi^{2}\right) \\
& =\varphi^{3 / 2}+\frac{3}{2} a_{1}^{1 / 2} c_{1}|x|^{-4}+o\left(|x|^{-4}\right) .
\end{aligned}
$$

We have used that from Lemma $34_{1} W(x)=c_{1}|x|^{-2}+o\left(|x|^{-2}\right)$. Hence (112) holds for $n=1$.

For $n \geqq 2$,

$$
\begin{aligned}
(\varphi+W)^{3 / 2}= & \left(\varphi_{n}+W_{n}\right)^{3 / 2}+\frac{3}{2}\left(\varphi_{n}+W_{n}\right)^{1 / 2}\left(\left(W-W_{n}\right)+\left(\varphi-\varphi_{n}\right)\right) \\
& +\left(\varphi_{n}+W_{n}\right)^{3 / 2} O\left(\left(\varphi-\varphi_{n}+W-W_{n}\right)^{2} /\left(\varphi_{n}+W_{n}\right)^{2}\right) \\
= & \left(\varphi_{n}+W_{n}\right)^{3 / 2}+\frac{3}{2} \varphi_{1}^{1 / 2}\left(\varphi-\varphi_{n}\right)+\frac{3}{2} a_{1}^{1 / 2} c_{n}|x|^{2 n-6} \\
& +o\left(|x|^{2 n-6}\right)+o\left(|x|^{4 n-10}\right),
\end{aligned}
$$

where we have again used Lemma 34 . Since $n \geqq 2,4 n-10 \geqq 2 n-6$, and we can forget about the last term.

On the other hand recalling that $\varphi_{1}^{3 / 2}=(4 \pi)^{-1} \Delta \varphi_{1}$ we find

$$
\left(\varphi-\tilde{\varphi}_{n}\right)^{3 / 2}+(4 \pi)^{-1} \Delta \tilde{\varphi}_{n}=(4 \pi)^{-1} \Delta \varphi_{n}+\frac{3}{2} \varphi_{1}^{1 / 2}\left(\varphi-\varphi_{n}\right)+o\left(|x|^{2 n-6}\right) .
$$

Equation (112) now follows from the identity

$$
\left(\varphi_{n}+W_{n}\right)^{3 / 2}-(4 \pi)^{-1} \Delta \varphi_{n}=\left(l_{n}-\frac{3}{2} a_{1}^{1 / 2} c_{n}\right)|x|^{2 n-6}+O\left(|x|^{2 n-4}\right) .
$$

Given $\varepsilon>0$, if $1 \leqq n \leqq 3$ define

$$
g_{ \pm}^{\varepsilon}(x)=\varphi(x)-\tilde{\varphi}_{n}(x)-\left(a_{n+1} \pm \varepsilon\right)|x|^{2 n-4},
$$

if $n=4$ define

$$
g_{ \pm}^{\varepsilon}(x)=\varphi(x)-\tilde{\varphi}_{n}(x)-\left(a_{n+1} \mp \varepsilon\right)|x|^{2 n-4} .
$$

Then writing $g_{ \pm}^{z}=g$ we find for $|x| \neq 0$,

$$
\begin{aligned}
& \Delta g-4 \pi g^{3 / 2}=4 \pi \psi^{2}-\Delta \tilde{\varphi}_{n}-4 \pi\left(\varphi-\tilde{\varphi}_{n}\right)^{3 / 2} \\
& \quad+\left(a_{n+1} \pm \varepsilon\right)\left(6 \pi a_{1}^{1 / 2}-(2 n-4)(2 n-3)\right)|x|^{2 n-6}+O\left(|x|^{2 n-4}\right) .
\end{aligned}
$$

Inserting (112) gives

$$
\Delta g-4 \pi g^{3 / 2}= \pm \varepsilon\left(6 \pi a_{1}^{1 / 2}-(2 n-4)(2 n-3)\right)|x|^{2 n-6}+o\left(|x|^{2 n-6}\right) .
$$


Recall that $\sigma,-\tau$ are the roots of $t(t+1)=18$, thus if $-\tau \leqq 2 n-3 \leqq \sigma$,i.e., $n \leqq 3$ then

$$
6 \pi a_{1}^{1 / 2}-(2 n-4)(2 n-3)=18-(2 n-4)(2 n-3)>0,
$$

and if $n=4$ this expression is negative. With the definitions (114) and (115) for $g_{ \pm}^{\varepsilon}$ it is now clear that we can choose $r_{\varepsilon}<R$ such that for all $x$ with $0<|x|<r_{\varepsilon}$,

$$
\Delta g_{-}^{\varepsilon}-4 \pi\left(g_{-}^{\varepsilon}\right)^{3 / 2}<0 \text { and } \Delta g_{+}^{\varepsilon}-4 \pi\left(g_{+}^{\varepsilon}\right)^{3 / 2}>0 .
$$

Now consider $\omega=\varphi_{1}+k|x|^{\sigma}=9 \pi^{-2}|x|^{-4}+k|x|^{\sigma}$. We know from (38) that for $|x| \neq 0, \Delta \omega(x) \leqq 4 \pi \omega(x)^{3 / 2}$. Choose $k=k_{\varepsilon} \geqq 0$ such that $\omega(x)>g_{+}^{\varepsilon}(x)$ for $|x|=r_{\varepsilon}$. From (101) $)_{1}$ we know that $g_{+}^{\varepsilon} / \omega \rightarrow 1$ as $x \rightarrow 0$. We will prove that

$$
\omega(x) \geqq g_{+}^{\varepsilon}(x)
$$

for all $x,|x| \leqq r_{\varepsilon}$. If not we must have $x_{0},\left|x_{0}\right|<r_{\varepsilon}$ such that

$$
\left(g_{+}^{\varepsilon} / \omega\right)\left(x_{0}\right)>1, \quad \nabla\left(g_{+}^{\varepsilon} / \omega\right)\left(x_{0}\right)=0 \quad \text { and } \quad \Delta\left(g_{+}^{\varepsilon} / \omega\right)\left(x_{0}\right)<0 .
$$

But then

$$
\begin{aligned}
\Delta g_{+}^{\varepsilon}\left(x_{0}\right) & =\omega \Delta\left(g_{+}^{\varepsilon} / \omega\right)\left(x_{0}\right)+2 \nabla \omega \nabla\left(g_{+}^{\varepsilon} / \omega\right)\left(x_{0}\right)+\left(g_{+}^{\varepsilon} / \omega\right) \Delta \omega\left(x_{0}\right) \\
& \leqq 4 \pi g_{+}^{\varepsilon} \omega\left(x_{0}\right)^{1 / 2} \leqq 4 \pi g_{+}^{\varepsilon}\left(x_{0}\right)^{3 / 2},
\end{aligned}
$$

which is in contradiction with (116). Hence (117) follows, i.e.,

$$
\varphi(x) \leqq \tilde{\varphi}_{n}(x)+\left(a_{n+1}+\varepsilon\right)|x|^{2 n-4}+\varphi_{1}(x)+k|x|^{\sigma}=\varphi_{n+1}(x)+\varepsilon|x|^{2 n-4}+O\left(|x|^{\sigma}\right),
$$

if $n \leqq 3$. If $n=4$ we get since $\sigma<4$,

$$
\begin{aligned}
\varphi(x) & \leqq \tilde{\varphi}_{4}(x)+\left(a_{n+1}-\varepsilon\right)|x|^{2 n-4}+\varphi_{1}(x)+k|x|^{\sigma} \\
& =\varphi_{5}(x)+O\left(|x|^{\sigma}\right)\left(=\varphi_{4}(x)+O\left(|x|^{\sigma}\right)\right) .
\end{aligned}
$$

To get similar lower bounds we use Lemma 21 with $r=r_{\varepsilon} . \quad V_{5}$ solves $\Delta V_{\zeta}=4 \pi V_{\zeta}^{3 / 2}$ for $|x| \neq 0$ and $V_{\zeta}\left(|x|=r_{\varepsilon}\right)=0$. Furthermore from Theorem 20 (iii) $\lim _{|x| \rightarrow 0}\left(|x| V_{\zeta}(x)\right)=\zeta<\infty$. Since $|x|^{4} g(x) \rightarrow 9 \pi^{-2}$ as $x \rightarrow 0$, we can of course assume that $g_{-}^{\varepsilon} \geqq 0$ for $|x| \leqq r_{\varepsilon}$. The set

$$
S_{\zeta}=\left\{x|0<| x \mid<r_{\varepsilon}, g_{-}^{\varepsilon}(x)<V_{\zeta}(x)\right\}
$$

therefore does not contain $\left\{|x|=r_{\varepsilon}\right\}$ nor points $x$ such that $|x|$ is small. An easy comparison argument shows that $S_{\zeta}=\emptyset$ for all $\zeta>0$. Taking $\zeta>\infty$ and using Lemma 21 gives

$$
9 \pi^{-2}|x|^{-4}+O\left(|x|^{\sigma}\right) \leqq g^{\varepsilon}-(x),
$$

or if $n \leqq 3$

$$
\varphi(x) \geqq \varphi_{n+1}(x)-\varepsilon|x|^{2 n-4}+O\left(|x|^{\sigma}\right) .
$$

If $n=4$ we get

$$
\varphi(x) \geqq \varphi_{5}(x)+O\left(|x|^{\sigma}\right)\left(=\varphi_{4}(x)+O\left(|x|^{\sigma}\right)\right) .
$$


End of Proof of Proposition 32. We have proved that (101)-(103) hold for $n=1$ and that if they hold for $n$ then (103) and (101) hold for $n+1$. Then clearly (102) also holds for $n+1$. We can then conclude that (101)-(103) hold for $n=6$. Since $3<\sigma<4$ this is equivalent to Proposition 32.

We can now reformulate Lemma 33 as

Corollary 36. $\nabla \varphi(x)=\nabla \varphi_{4}(x)+O\left(|x|^{\sigma-1}\right)$ and $\nabla \psi(x)=\nabla \psi_{4}(x)+O\left(|x|^{\sigma}\right)$.

\section{Uniqueness}

The proofs of the main Theorems 1-7 that are given in Sect. 8 below will be simple consequences of the following theorem and its proof.

Theorem 37. (a) Given $\mu \geqq 0$, there exist unique $\varphi, 0<\psi$ and $Q \in \mathbb{R}$, with $\varphi \in L_{\text {loc }}^{2+\delta}\left(\mathbb{R}^{3} \backslash\left\{\mathscr{R}_{1}, \ldots, \mathscr{R}_{L}\right\}\right)$ for some $\delta>0$, and $\psi$ in $H^{1}$ of the complement of any compact neighborhood of $\left\{\mathscr{R}_{1}, \ldots, \mathscr{R}_{L}\right\}$ and such that $\varphi, \psi$, and $Q$ satisfy (85)-(87).

(b) Let $Q_{\infty}$ be the value of $Q$ for $\mu=0$ as described in (a). Then given $Q \in\left(-\infty, Q_{\infty}\right)$, there exist unique $\varphi, 0<\psi$ and $\mu \in \mathbb{R}$, with $\varphi$ and $\psi$ as in (a) such that $\varphi, \psi$, and $\mu$ satisfy (85)-(87).

The existence parts of (a) and (b) follow from Proposition 31. The uniqueness parts will follow from a series of lemmas which are of independent interest.

\section{Lemma 38.}

$$
\varphi, \psi \in C^{\infty}\left(\mathbb{R}^{3} \backslash\left\{\mathscr{R}_{1}, \ldots, \mathscr{R}_{\mathbf{K}}\right\}\right) \text { and } \psi \in L_{\mathrm{loc}}^{\infty}\left(\mathbb{R}^{3} \backslash\left\{\mathscr{R}_{1}, \ldots, \mathscr{R}_{L}\right\}\right) .
$$

Proof. This is standard elliptic regularity.

We can now conclude from Proposition 32 that for $j=1, \ldots, L, \varphi$ and $\psi$ satisfy the following asymptotic expansions near $\mathscr{R}_{j}$ :

$$
\varphi(x)-\mu=\varphi_{4}\left(x-\mathscr{R}_{j}\right)+O\left(\left|x-\mathscr{R}_{j}\right|^{\sigma}\right) \quad \text { and } \quad \psi(x)=\psi_{4}\left(x-\mathscr{R}_{j}\right)+O\left(\left|x-\mathscr{R}_{j}\right|^{\sigma+1}\right) .
$$

The idea is now to subtract the singular part from $\varrho=\psi^{2}$. We define the renormalized density (see also (23))

$$
\varrho^{(2)}(x)=\psi(x)^{2}-\left\{\sum_{j=1}^{L} b_{1}^{2}\left|x-\mathscr{R}_{j}\right|^{-6}+2 b_{1} b_{2}\left|x-\mathscr{R}_{j}\right|^{-4}\right\},
$$

where the $b$ 's are as in the previous section. From Proposition 32 we know that $\varrho^{(2)}(x)=O\left(\left|x-\mathscr{R}_{j}\right|^{-2}\right)$. In fact we can conclude more. From (93) we see that

$$
\Delta \varphi_{4}(x)=4 \pi \psi_{4}(x)^{2}+O\left(|x|^{2}\right),
$$

thus $\psi_{4}(x)^{2}$ contains no term of order $|x|^{-2}$ (we could have concluded this from the numerical values of the $b_{n}$ 's also). Hence $\varrho^{(2)}(x)=O(1)$ or

$$
\varrho^{(2)} \in L_{\text {loc }}^{\infty}\left(\mathbb{R}^{3}\right) \cap L^{1}\left(\mathbb{R}^{3}\right)
$$

Notice that $\varrho^{(2)}$ is not necessarily everywhere positive, but we can think of it as a charge density. $\varrho^{(2)}$ has the following remarkable property: 


\section{Lemma 39.}

$$
\int \varrho^{(2)}(x) d x=Q+\sum_{j=L+1}^{K} z_{j}
$$

Proof. It is clear from (93) that $\Delta \varphi_{2}=4 \pi\left(b_{1}^{2}|x|^{-6}+2 b_{1} b_{2}|x|^{-4}\right)$. Hence if we define

$$
\varphi^{(2)}(x)=\varphi(x)-\left\{\sum_{j=1}^{L} a_{1}\left|x-\mathscr{R}_{j}\right|^{-4}+a_{2}\left|x-\mathscr{R}_{j}\right|^{-2}\right\},
$$

we get

$$
\Delta \varphi^{(2)}(x)=4 \pi\left\{\varrho^{(2)}(x)-\sum_{j=L+1}^{K} z_{j} \delta\left(x-\mathscr{R}_{j}\right)\right\},
$$

for $x \notin\left\{\mathscr{R}_{1}, \ldots, \mathscr{R}_{L}\right\}$. From Proposition $32 \varphi^{(2)} \in L_{\text {loc }}^{2}\left(\mathbb{R}^{3}\right)$. Thus $\Delta \varphi^{(2)} \in H_{\text {loc }}^{-2}\left(\mathbb{R}^{3}\right)$. Since the Dirac-measure $\delta_{0} \notin H^{-3 / 2}\left(\mathbb{R}^{3}\right)$ we see that $\partial^{\alpha} \delta_{0} \notin H^{-2}\left(\mathbb{R}^{3}\right)$, for all multiindices $\alpha$ with $|\alpha| \geqq 1$. We can thus conclude from (122) that there exist $z_{j} \in \mathbb{R}$, $j=1, \ldots, L$ such that on all of $\mathbb{R}^{3}$,

$$
\Delta \varphi^{(2)}(x)=4 \pi\left\{\varrho^{(2)}(x)-\sum_{j=1}^{K} z_{j} \delta\left(x-\mathscr{R}_{j}\right)\right\}
$$

or

$$
\Delta\left\{\varphi^{(2)}(x)+\sum_{j=1}^{L} z_{j}\left|x-\mathscr{R}_{j}\right|^{-1}\right\}=4 \pi \varrho^{(2)}(x)-\sum_{j=\boldsymbol{L}+1}^{K} z_{j} \delta\left(x-\mathscr{R}_{j}\right) .
$$

Now the right-hand side is in $L^{2}$ near $\mathscr{R}_{j}, j=1, \ldots, L$. Hence

$$
\varphi^{(2)}(x)+\sum_{j=1}^{L} z_{j}\left|x-\mathscr{R}_{j}\right|^{-1}
$$

is in $H^{2}$ near $\mathscr{R}_{j}$. But from Proposition $32 \varphi^{(2)}$ is bounded near $\mathscr{R}_{j}, j=1, \ldots, L$. We conclude that $z_{j}=0$ for $j=1, \ldots, L$. Thus $(122)$ holds on all of $\mathbb{R}^{3}$. It is now clear that

$$
h(x)=\varphi^{(2)}(x)-\left\{\sum_{j=L+1}^{K} z_{j}\left|x-\mathscr{R}_{j}\right|^{-1}-\varrho^{(2)} *|x|^{-1}\right\}
$$

is harmonic and $h(x) \rightarrow 0$ as $|x| \rightarrow \infty$. Hence $h(x)=0$, i.e.,

$$
\varphi^{(2)}(x)=\sum_{j=L+1}^{K} z_{j}\left|x-\mathscr{R}_{j}\right|^{-1}-\varrho^{(2)} *|x|^{-1} .
$$

From this and (87) we find

$$
Q=-\lim _{x \rightarrow \infty}\left(\varphi^{(2)}(x)|x|\right)=\int \varrho^{(2)}(x) d x-\sum_{j=L+1}^{K} z_{j}
$$

We denote

$$
\begin{aligned}
W(x)=\psi^{4 / 3} & -\sum_{j=1}^{L}\left(a_{1}\left|x-\mathscr{R}_{j}\right|^{-4}+a_{2}\left|x-\mathscr{R}_{j}\right|^{-2}\right) \\
& -\sum_{j=L+1}^{K} z_{j}\left|x-\mathscr{R}_{j}\right|^{-1}+\varrho^{(2)} *|x|^{-1}+\mu .
\end{aligned}
$$


From (121) and (123) we find

Lemma 40. $\psi$ solves the Renormalized TFW-equation

on $\mathbb{R}^{3} \backslash\left\{\mathscr{R}_{1}, \ldots, \mathscr{R}_{L}\right\}$.

$$
-\Delta \psi+W \psi=0
$$

Lemma 41. $W$ is bounded at infinity.

Proof. For the solutions constructed in Proposition 31 we of course know that $W \rightarrow \mu$ as $|x| \rightarrow \infty$. For the abstract solution we just have to prove that $\psi$ is bounded at infinity. This is not too difficult and is left to the reader.

We define the following functional:

$$
L(f)=\int_{\mathbb{R}^{3}}|\nabla f|^{2}+W|f|^{2} d x,
$$

for all functions in the set

$$
F=\left\{f \in H^{1}\left(\mathbb{R}^{3}\right) \mid f=O\left(\left|x-\mathscr{R}_{j}\right|^{\sigma+1}\right), \nabla f=O\left(\left|x-\mathscr{R}_{j}\right|^{\sigma}\right), \text { for } j=1, \ldots, L\right\} .
$$

Notice that $L(f)<\infty$ for all $f \in F$.

Lemma 42. $f \in F \Rightarrow L(f) \geqq 0$.

Proof. It is enough to consider $f \in C_{0}^{\infty}\left(\mathbb{R}^{3} \backslash\left\{\mathscr{R}_{1}, \ldots, \mathscr{R}_{L}\right\}\right)$. Then

$-\int W|f|^{2}=-\int(\Delta \psi / \psi)|f|^{2} \leqq-\int \psi^{-2}(\nabla \psi)^{2}|f|^{2}+2 \int \psi^{-1} \nabla|\psi||f||\nabla f| \leqq \int|\nabla f|^{2}$.

Lemma 43. If $f \in F$ then

$$
L(f)=\int_{\mathbb{R}^{3}}\left(|\nabla(\psi+f)|^{2}-|\nabla \psi|^{2}+W|\psi+f|^{2}-W \psi^{2}\right) d^{3} x .
$$

Proof. The right-hand side of (128) is equal to

$$
L(f)+\operatorname{Re}\left\{\int_{\mathbb{R}^{3}} 2 \nabla f \nabla \psi+2 f \Delta \psi\right\} .
$$

Notice that

$$
|\nabla f||\nabla \psi|+|f||\Delta \psi|=O\left(\left|x-\mathscr{R}_{j}\right|^{\sigma-4}\right)+|W||f||\psi|=O\left(\left|x-\mathscr{R}_{j}\right|^{\sigma-4}\right),
$$

hence is in $L^{1}\left(\mathbb{R}^{3}\right)$. We just have to argue that we can integrate by parts. But this is clear from $|\nabla \psi||f|=O\left(\left|x-\mathscr{R}_{j}\right|^{\sigma-3}\right)$.

We can now give the

Proof of Theorem 37 (a). We have to prove the uniqueness. Assume $(\varphi, \psi, Q)$ and $(\tilde{\varphi}, \tilde{\psi}, \widetilde{Q})$ are two different triples satisfying (85)-(87) for the same $\mu$. We have two corresponding functionals $L, \tilde{L}$. Notice that from Proposition 32 and Corollary 36 $\psi-\tilde{\psi} \in F$. Thus from Lemmas 42 and 43 ,

$$
\begin{aligned}
0 & \leqq L(\tilde{\psi}-\psi)+L(\psi-\tilde{\psi})=\int(W-\tilde{W})\left(\tilde{\psi}^{2}-\psi^{2}\right) d x \\
& =\int\left(\psi^{4 / 3}-\tilde{\psi}^{4 / 3}\right)\left(\tilde{\psi}^{2}-\psi^{2}\right)+\left(\varrho^{(2)}-\tilde{\varrho}^{(2)}\right) *|x|^{-1}\left(\tilde{\psi}^{2}-\psi^{2}\right) d x \\
& \leqq \int\left(\varrho^{(2)}-\tilde{\varrho}^{(2)}\right) *|x|^{-1}\left(\tilde{\varrho}^{(2)}-\varrho^{(2)}\right) d x .
\end{aligned}
$$


Since $|x-y|^{-1}$ is a positive definite kernel this last expression is non-negative if and only if $\tilde{\psi}=\psi$. From (121) and (123) we find $\tilde{\varphi}=\varphi . \widetilde{Q}=Q$ follows from (87).

Proof of Theorem 37 (b). (Existence) From Theorem 37 (a) and Proposition 31 we get that $Q_{\infty}=\lim _{z^{\prime} \rightarrow \infty} Q_{c}(z)>0$ (the lower bound is a consequence of Theorem 26). The existence then follows from Proposition 31, since if $Q<Q_{\infty}$, then for $z_{1}, \ldots, z_{L}$ large enough $Q<Q_{c}(z)$.

For the uniqueness we proceed as in case (a). Given two triples $(\varphi, \psi, \mu)$ and $(\tilde{\varphi}, \tilde{\psi}, \tilde{\mu})$ satisfying $(85)-(87)$ for the same $Q$, then

$$
\begin{aligned}
0 \leqq & \int\left(\psi^{4 / 3}-\tilde{\psi}^{4 / 3}\right)\left(\tilde{\psi}^{2}-\psi^{2}\right)+\left(\varrho^{(2)}-\tilde{\varrho}^{(2)}\right) *|x|^{-1}\left(\tilde{\varrho}^{(2)}-\varrho^{(2)}\right) d x \\
& +(\mu-\tilde{\mu}) \int\left(\psi^{2}-\tilde{\psi}\right) d x .
\end{aligned}
$$

But from Lemma 39

$$
\int \psi^{2}-\tilde{\psi}^{2} d x=\int \varrho^{(2)}-\tilde{\varrho}^{(2)} d x=0 .
$$

We again conclude $\tilde{\psi}=\psi, \tilde{\varphi}=\varphi . \tilde{\mu}=\mu$ follows from (85).

\section{Proofs of Main Theorems}

As a simple consequence of the results of the previous section we can now give

Proof of Theorem 1 and Theorem 4. It follows from Proposition 31 that any sequence has a subsequence satisfying Theorem 1. From Theorem 37 all the subsequences must have the same limit. This implies Theorem 1 . For Theorem 4 , if $Q \in\left(-\infty, Q_{\infty}\left(\underline{z}^{\prime \prime}, \mathscr{R}\right)\right)$, then it follows from Theorem 1 that $Q<Q_{c}(\underline{z})$ for $\underline{z}^{\prime}$ large enough. Then Theorem 4 follows in the same way as Theorem 1.

Proof of Theorem 2. As noted in the remark after the theorem this is just a special case of Theorem 1, except for the lower bound which follows from Theorem 26.

Proof of Corollary 3. We first note that $Z \mapsto R_{Z}$ is continuous. This easily follows from Theorem 6 in [2]. If $R_{Z}$ is not convergent we can find sequences converging to any value in some open interval. Since $\varphi_{Z}^{\prime}\left(R_{Z}\right)=0$ we would conclude that the limit $\varphi_{\mu=0}$ is constant in an open set. But then $\psi_{\mu=0}$ is zero on this set since $\Delta \varphi_{\mu=0}$ $=4 \pi \psi_{\mu=0}^{2}$.

Proof of Theorem 6 (a) and (b). This is just Proposition 32. Theorem 6 (b) is a consequence of the exponential decay (77) and (86) and the TFW-equation.

Proof of Theorem 7. That $\psi_{\mu}$ has the properties given follows from Lemma 40 and Lemma 39. Equation (25) is just (123). The uniqueness follows from Theorem 37 and its proof, if we can prove that $\nabla \psi_{\mu}$ is in $L^{2}$ on the complement of any compact neighborhood of $\left\{R_{1}, \ldots, \mathscr{R}_{L}\right\}$. But this is clear, since from (24) $\Delta \psi_{\mu}$ is in $L^{2}$ over such a set.

Acknowledgements. I would like to thank Elliott Lieb for suggesting the problems studied in this work and for many helpful discussions. Thanks also to Rafael Benguria and Heinz Siedentop for their interest and encouragement. 


\section{References}

1. Benguria, R., Brezis, H., Lieb, E.H.: The Thomas-Fermi-von Weizsäcker theory of atoms and molecules. Commun. Math. Phys. 79, 167-180 (1981)

2. Benguria, R., Lieb, E.H.: The most negative ion in the Thomas-Fermi-von Weizsäcker theory of atoms and molecules. J. Phys. B 18, 1045-1059 (1985)

3. Brezis, H., Lieb, E.H.: Long range potentials in Thomas-Fermi theory. Commun. Math. Phys. 65, 231-246 (1979)

4. Dreizler, R.M.: Private communication

5. Gagliardo, E.: Ulteriori propieta di alcune classi di funzioni in piu variabili. Ric. Mat. 8, 24-51 (1959)

6. Hoffmann-Ostenhof, $\mathrm{T}$ : A comparison theorem for differential inequalities with applications in quantum mechanics. J. Phys. A 13, 417-424 (1980)

7. Hughes, W.: An atomic energy lower bound that gives Scott's correction. PhD thesis, Princeton, Department of Mathematics, 1986

8. Liberman, D.A., Lieb, E.H.: Numerical calculation of the Thomas-Fermi-von Weizsäcker function for an infinite atom without electron repulsion. Los Alamos National Laboratory Report, LA 9186-MS, 1981

9. Lieb, E.H.: Bound on the maximum negative ionization of atoms and molecules. Phys. Rev. A 29, 3018-3028 (1984)

10. Lieb, E.H.: Thomas-Fermi and related theories of atoms and molecules. Rev. Mod. Phys. 53, $603-640(1981)$

11. Lieb, E.H., Simon, B.: The Thomas-Fermi theory of atoms, molecules, and solids. Adv. Math. 23, 22-116 (1977)

12. Nirenberg, L.: On elliptic partial differential equations. Ann. Scu. Norm. Sup. Pisa 13, 115-162 (1959)

13. Rother, W.: Zur Thomas-Fermi-von Weizsäcker Theorie für Atome und Moleküle. Bayreuther Mathematische Schriften 18, 39-145 (1985)

14. Seco, L.A., Sigal, I.M., Solovej, J.P.: Bound on the ionization energy of large atoms. Commun. Math. Phys. (to appear)

15. Siedentop, H., Weikard, R.: On the leading correction of the Thomas-Fermi model: Lower bound - with an appendix by A.M.K. Müller. Invent. Math. 97, 159-193 (1989)

16. Siedentop, H., Weikard, R.: On the leading energy correction for the statistical model of the atom: interacting case. Commun. Math. Phys. 112, $471-490$ (1987)

17. Solovej, J.P.: Universality in the Thomas-Fermi-von Weizsäcker Theory of Atoms and Molecules. PhD thesis, Princeton, Department of Mathematics, June 1989

18. Sommerfeld, A.: Asymptotische Integration der Differentialgleichung des ThomasFermischen Atoms. Z. Phys. 78, 283-308 (1932)

19. Stich, W., Gross, E.K.U., Malzacher, P., Dreizler, R.M.: Accurate solution of the ThomasFermi-Dirac-Weizsäcker variational equations for the case of neutral atoms and positive ions. Z. Phys. A 309, 5-11 (1982)

20. Veron, L.: Singular solutions of some nonlinear elliptic equations. Nonlinear Analysis 5, 225-242 (1981)

Communicated by B. Simon

Received August 16, 1989 